This document is the Accepted Manuscript version of a Published Work that appeared in final form in Macromolecules copyright (C) 2018 American Chemical Society after peer review and technical editing by the publisher.

To access the final edited and published work see https://doi.org/10.1021/acs.macromol.7b02638

\title{
Influence of chain topology (cyclic versus linear) on the nucleation and isothermal crystallization of poly(L-lactide) and poly(D-lactide)
}

\author{
${ }^{1}$ Nerea Zaldua, ${ }^{2,3}$ Romain Liénard, ${ }^{2}$ Thomas Josse, ${ }^{4}$ Manuela Zubitur, ${ }^{1}$ Agurtzane \\ Mugica, ${ }^{5}$ Amaia Iturrospe, ${ }^{5}$ Arantxa Arbe, ${ }^{3}$ Julien De Winter, ${ }^{2}$ Olivier Coulembier*, \\ ${ }^{1,6}$ Alejandro J. Müller*
}

${ }^{1}$ POLYMAT and Polymer Science and Technology Department, Faculty of Chemistry, University of the Basque Country UPV/EHU, Paseo Manuel de Lardizabal 3, 20018 Donostia-San Sebastián, Spain.

${ }^{2}$ Laboratory of Polymeric and Composite Materials, University of Mons - UMONS, Place du Parc 23, 7000 Mons, Belgium.

${ }^{3}$ Organic Synthesis and Mass Spectrometry Laboratory, University of Mons - UMONS, Place du Parc 23, 7000 Mons, Belgium.

${ }^{4}$ Chemical and Environmental Engineering Department, Polytechnic School, University of the Basque Country UPV/EHU, 20018 Donostia-San Sebastián, Spain.

${ }^{5}$ Centro de Física de Materiales (CFM) (CSIC-UPV/EHU) - Materials Physics Center (MPC), Paseo Manuel de Lardizabal 5, 20018 Donostia-San Sebastián, Spain.

${ }^{6}$ IKERBASQUE, Basque Foundation for Science, Bilbao, Spain

*Corresponding authors. E-mails: alejandrojesus.muller@ehu.es, olivier.coulembier@umons.ac.be 


\begin{abstract}
In this paper, ring closure click chemistry methods have been used to produce cyclic $c$-PLLA and $c$-PDLA of a number average molecular weight close to $10 \mathrm{~kg} / \mathrm{mol}$. The effects of stereochemistry of the polymer chains and their topology on their structure, nucleation and crystallization were studied in detail employing Wide Angle X-ray Scattering (WAXS), Small Angle X-ray Scattering (SAXS), Polarized Light Optical Microscopy (PLOM) and standard and advanced Differential Scanning Calorimetry (DSC). The crystal structures of linear and cyclic PLAs are identical to each other and no differences in superstructural morphology could be detected. Cyclic PLA chains are able to nucleate much faster and to produce a higher number of nuclei in comparison to linear analogues, either upon cooling from the melt or upon heating from the glassy state. In the samples prepared in this work, a small fraction of linear or higher molecular weight cycles was detected (according to SEC analyses). The presence of such "impurities" retards spherulitic growth rates of $c$-PLAs making them nearly the same as those of $l$-PLAs. On the other hand, the overall crystallization rate determined by DSC was much larger for $c$-PLAs, as a consequence of the enhanced nucleation that occurs in cyclic chains. The equilibrium melting temperatures of cyclic chains were determined and found to be $5{ }^{\circ} \mathrm{C}$ higher in comparison with values for $l$-PLAs. This result is a consequence of the lower entropy of cyclic chains in the melt. Self-nucleation studies demonstrated that $c$-PLAs have a shorter crystalline memory than linear analogues, as a result of their lower entanglement density. Successive selfnucleation and annealing (SSA) experiments reveal the remarkable ability of cyclic molecules to thicken, even to the point of crystallization with extended collapsed ring conformations. In general terms, stereochemistry had less influence on the results obtained in comparison with the dominating effect of chain topology.
\end{abstract}

Keywords: Cyclic polymers, nucleation kinetics, crystallization kinetics, equilibrium melting point. 


\section{Introduction}

Cyclic polymers are remarkable materials that differ from their linear analogues in their lack of chain ends. However, this seemingly small topological peculiarity can lead to dramatic differences in chain conformation and therefore in polymer properties.

The absence of chain ends could be an advantage in some applications, since circular topologies are designed to prevent any possible reaction or interaction across the ends of the chain. The interest generated in these materials has increased as cyclic structures have unique and improved properties. ${ }^{1}$ However, the difficulty in the synthesis of cyclic polymers with high cycling and purification efficiency has led to a lack of understanding of the properties. ${ }^{1}$

The crystallization of cyclic polymers is a complex subject that needs more research to understand all the relevant factors involved. ${ }^{2}$ The methods of synthesis and purification used in the production of cyclic polymers with high purity have evolved for decades. Today, new approaches to synthesis have allowed the preparation of a wide range of high-purity cyclic polymers as well as more complex cyclic topologies. ${ }^{3-11}$ These new developments have allowed researchers to study the differences between properties of the cyclic and linear material, such as the glass transition temperature, ${ }^{12,13}$ melt viscosity and diffusion, ${ }^{12-15}$ morphology ${ }^{16-18}$ and crystallization. ${ }^{19-32}$

In the particular case of crystallization, several literature reports on cyclic polymers indicate different trends on the results and therefore, a universal interpretation of the lack of chain ends for all polymers is not possible. The crystallization of cyclic chains depends on the type of material used, and on the level of purity, among other factors. The reported trends in the literature can be arbitrarily divided into 3 groups. ${ }^{2,28}$

- In group 1 are encompassed the cyclic polymers reported to have the highest nucleation density, lower equilibrium melting points $\left(T_{m}^{0}\right)$ and lower spherulitic growth rates $(G)$ than linear polymers of similar molecular weights. The reported polymers in this group are polyethylene (PE) by Kitahara et al. ${ }^{24}$ and poly(tetrahydrofurans) (PTHF)s by Tezuka et al. ${ }^{20}$ and Takeshita et al. ${ }^{32}$

- Group 2 contains studies of cyclic oligomeric poly(oxyethylene) (POE) reported by $\mathrm{Yu}$ et al. ${ }^{19}$, Cooke et al. ${ }^{18}$ and Nam et al. ${ }^{14}$, which showed lower melting points, higher value of $G$ and the same values of $T_{m}^{0}$ than their linear equivalents. Poly( $\varepsilon$-caprolactone) from Li et al. ${ }^{33}$ has also been included in this group. 
- Finally, group 3 includes works where cyclic polymers have been found to have higher values of crystallization temperature $\left(T_{c}\right)$, higher melting point values $\left(T_{m}\right)$ and/or higher values of $G$ and/or higher values of overall crystallization rate $\left(1 / \tau_{50 \%}\right)$. This group contains oligomeric alkanes, reported by Lee and Wegner et al., ${ }^{34}$ PE-s of Bielawski et al. ${ }^{35}$ and PCL studies published by Schäler et al. ${ }^{22}$ and Müller et al. .1,23,27,28,29,36 $^{2}$

In the present work, linear and cyclic polylactides have been employed. The influence of the lack of chain ends on the thermal properties and crystallization of cyclic PLA has not been studied in detail. Shin et al. ${ }^{26}$ in 2012, prepared mixtures of PLLA and (PDLA) with $\mathrm{M}_{n}$ of 15 and $19 \mathrm{~kg} / \mathrm{mol}$ with cyclic and linear topologies, and characterized them by small-angle X-ray scattering (SAXS). They observed that the lamellar thickness and long period of cyclic PLLA lamellae are approximately $20 \%$ larger than those of linear PLLA. They obtained $20 \mathrm{~nm}$ long period for cyclic PLLA, which is in the order of the length of the extended chain ( $37 \mathrm{~nm}$ considering a $10_{3}$ helix). The authors attributed the high value of the lamellar thicknesses and long period of the cyclic PLLA relative to the linear PLLA to a possible topological restriction for folding of cyclic PLLA chains. However, in spite of the differences in lamellar thickness, the $T_{m}$ values reported were 150 and $135^{\circ} \mathrm{C}$ for linear and cyclic PLLA, respectively. These results were not explained by Shin et al.

In contrast, Sugai et al. ${ }^{31}$ studied cyclic PLAs and reported smaller lamellar thickness values as compared to linear chains. The authors attributed the results to the more compact conformations of the cyclic chains. They also used an unmodified Thomson-Gibbs equation to determine surface free energy $\left(\sigma_{e}\right)$ of their samples. They obtained a lower free interfacial energy $\left(\sigma_{e}\right)$ for the cyclic PLLA than for the linear analogue. According to the authors, this result suggested that cyclic PLLA forms a wellordered loop structure on the crystal interface and amorphous state, whereas linear PLLA has chain ends that are probably not so organized as in the cyclic ones. In another study, Sugai et al. ${ }^{37}$ reported that the change of the topology between cyclic and linear has effects on the melting peaks with lower melting temperatures in the cyclic topology. They reported values of $151^{\circ} \mathrm{C}$ for linear and $147^{\circ} \mathrm{C}$ for the cyclic analogues.

However, as far as the authors are aware, the kinetics of nucleation, spherulitic growth and overall crystallization for cyclic and linear PLA has not been reported in the literature so far. In the present work, we have analyzed the effect of the cyclic and linear topology on the thermal properties and crystallization kinetics. Additionally, we have 
applied advanced DSC techniques to study the effect of chain topology on the selfnucleation behavior and SSA (Successive Self-Nucleation and Annealing) thermal fractionation of the samples.

\section{Materials}

L- and D-lactide (L-,D-LA) ( $\geq 99 \%)$ were purchased from Galactic, recrystallized from dried toluene three times and stored in a glove box under dry nitrogen atmosphere $\left(\mathrm{O}_{2}<5 \mathrm{ppm}, \mathrm{H}_{2} \mathrm{O}<1 \mathrm{ppm}\right)$ prior to use. Reagents materials were purchased from Aldrich and used without further purification, unless otherwise noted. 1,8-Diazabicyclo[5.4.0] undec-7-ene (DBU) was dried over $\mathrm{BaO}$, distilled and stored in a glove box. Copper (I) bromide was purified by washing with acetic acid. 11-Azido-1undecanol was synthesized as reported in the literature and dried over anhydrous $\mathrm{MgSO}_{4}$ prior storage in the glovebox. ${ }^{38} \mathrm{~N}, \mathrm{~N}, \mathrm{~N}$, N",N"-Pentamethyldiethylenetriamine (PMDETA) was dried over $\mathrm{MgSO}_{4}$ prior storage in the glovebox. 4-Pentynoic anhydride was synthesized as reported in the literature. ${ }^{39}$ Tetrahydrofuran (THF, Labscan, 99\%) was dried using a MBraun solvent purification system under $\mathrm{N}_{2}$.

\section{Synthesis procedures}

\section{Synthesis of linear PL(D)LA precursors}

Ring-opening polymerization (ROP) was applied to synthesize PL(D)LA samples with $\omega$-hydroxyl functionality. In a glovebox under nitrogen atmosphere, a vial was charged with L(D)-lactide (1.00 g, $\left.6.910^{-3} \mathrm{~mol}\right)$ and 11-azido-1-undecanol (20 $\mu \mathrm{L}$; $\left.9.410^{-5} \mathrm{~mol}\right)$ initiator, in dichloromethane $(7.5 \mathrm{~mL})$. After solubilization, 1,8diazabicyclo [5.4.0] undec-7-ene (DBU) (14.5 $\left.\mu \mathrm{L} ; 9.510^{-5} \mathrm{~mol}\right)$ catalyst was added into the medium. After 9 minutes under stirring, the polymerization was quenched with benzoic acid. The homopolymer was recovered by precipitation into cold methanol to give $\alpha$-azide $\omega$-hydroxy PL(D)LA. ${ }^{40,41}$

Finally, the linear $\alpha$-azide $\omega$-hydroxy PL(D)LA homopolymers were functionalized on the $\omega$ chain end with pentyonic anhydride leading to $\alpha$-azide $\omega$-alkyne PL(D)LA $\left(l\right.$-PLLA: $\quad \mathrm{M}_{\mathrm{n}}{ }^{\mathrm{SEC}, \text { app }}=16700 \mathrm{~g} / \mathrm{mol}, \mathrm{M}_{\mathrm{n}}{ }^{\mathrm{MALDI}}=9700 \mathrm{~g} / \mathrm{mol}, \bigoplus_{\mathrm{M}} 1.10 ; l$ PDLA: $\left.\mathrm{M}_{\mathrm{n}}{ }^{\mathrm{SEC}, \text { app }}=16200 \mathrm{~g} / \mathrm{mol}, \mathrm{M}_{\mathrm{n}}{ }^{\mathrm{MALDI}}=9500 \mathrm{~g} / \mathrm{mol}, \bigoplus_{\mathrm{M}} 1.11\right){ }^{42}$ 


\section{Preparation of cyclic PL(D)LA}

The CuAAc reaction was performed in pseudo-high-dilution conditions by dropwise addition of linear PL(D)LA precursor. In a glovebox under nitrogen pressure $\left(\mathrm{O}_{2}<5\right.$ ppm, $\left.\mathrm{H}_{2} \mathrm{O}<1 \mathrm{ppm}\right)$, a solution of $\alpha$-azido- $\omega$-alkyne PLLA (25 mg; $2.810^{-6} \mathrm{~mol}$; 1 equivalent) in THF ( $1 \mathrm{~mL})$ was injected over 50 minutes in a solution of $\mathrm{Cu}(\mathrm{I}) \mathrm{Br}(48$ $\mathrm{mg} ; 120$ equivalents) and pentamethyldiethylenetriamine (PMDETA) (140 $\mu \mathrm{L} ; 240$ equivalents) in THF ( $6 \mathrm{~g} ; 6.7 \mathrm{~mL})$ under stirring using a repetitive pipette. The solvent was evaporated before solubilization of the crude product in $\mathrm{CH}_{2} \mathrm{Cl}_{2}$. Then, the medium was extracted two times using a saturated $\mathrm{NaHSO}_{4}$ aqueous solution. The organic phase was dried over $\mathrm{MgSO}_{4}$, filtered and concentrated by evaporation prior to precipitation into heptane. The preparation procedure was the same for cyclo PDLA (c-PLLA: $\mathrm{M}_{\mathrm{n}}{ }^{\mathrm{SEC}, \text { app }}(c$-PLLA $)=14000 \mathrm{~g} / \mathrm{mol}, \mathrm{M}_{\mathrm{n}}{ }^{\mathrm{MALDI}}=10000 \mathrm{~g} / \mathrm{mol}, \bigoplus_{\mathrm{M}} 1.21 ; c$-PDLA: $\left.\mathrm{M}_{\mathrm{n}}{ }^{\mathrm{SEC}, \text { app }}=13900 \mathrm{~g} / \mathrm{mol}, \mathrm{M}_{\mathrm{n}}{ }^{\mathrm{MALDI}}=9600 \mathrm{~g} / \mathrm{mol}, \bigoplus_{\mathrm{M}} 1.22\right)$. The synthesis procedure is summarized in Scheme 1.

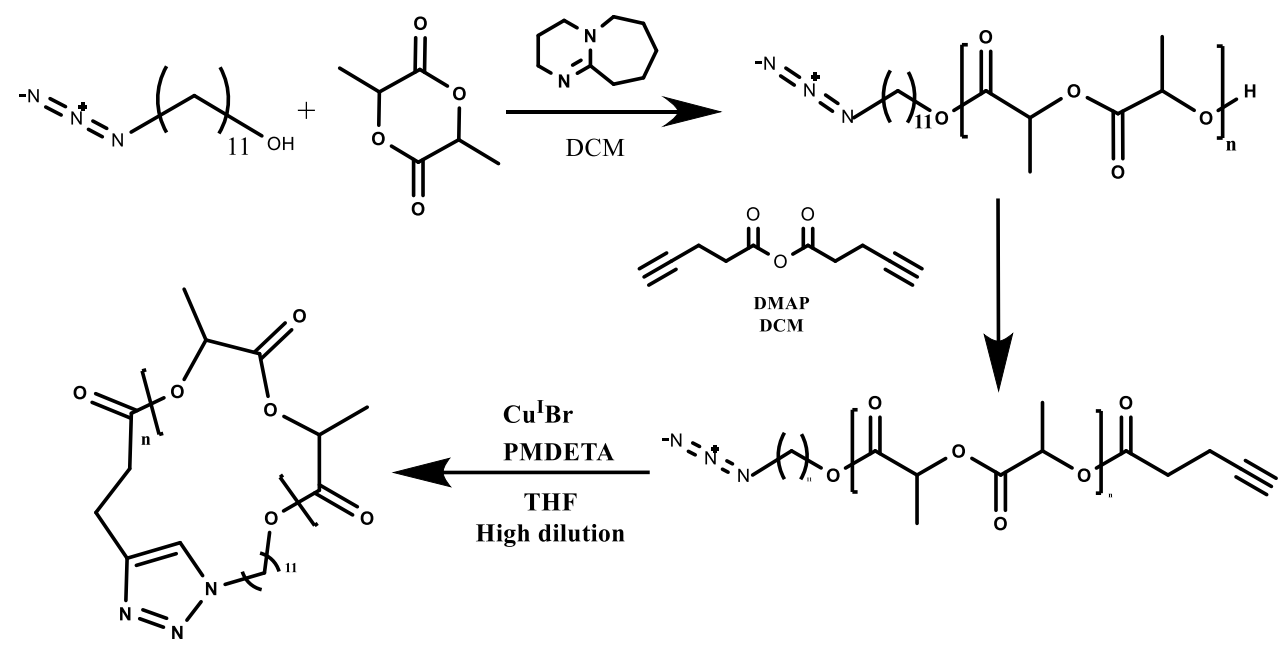

Scheme 1. Synthesis pathway applied to the generation of cyclo $P L(D) L A$.

\section{Characterization}

${ }^{1} \mathrm{H}$ NMR spectra were obtained on a Bruker AMX500 (500MHz) spectrometer at $25{ }^{\circ} \mathrm{C}$ in $\mathrm{CDCl}_{3}(20 \mathrm{mg} / 0.6 \mathrm{~mL})$. Size exclusion chromatography (SEC) was performed in THF (with 2 vol\% of triethylamine added) at $35^{\circ} \mathrm{C}$ using a Polymer Laboratories liquid chromatograph equipped with a PL-DG802 degasser, an isocratic HPCL pump LC 1120 (flow rate $=1 \mathrm{~mL} / \mathrm{min}$ ), a Marathon autosampler (loop volume $=200 \mu \mathrm{L}$, 
solution conc. $=1 \mathrm{mg} / \mathrm{min}$ ), a PL-DRI refractive index detector and three columns: a PL gel $10 \mu \mathrm{m}$ guard column and two PL gel Mixed-B $10 \mu \mathrm{m}$ columns (linear columns for separation of MW $\mathrm{MS}$ ranging from 500 to $10^{6}$ Daltons). Polystyrene standards were used for calibration.

Positive-ion MALDI-MS experiments were conducted using a Waters QTOF Premier mass spectrometer equipped with a Nd:YAG laser (third harmonic) operating at $355 \mathrm{~nm}$ with a maximum output of $65 \mu \mathrm{J}$ delivered to the sample in $2.2 \mathrm{~ns}$ pulses at 50 $\mathrm{Hz}$ repeating rate. Time of flight mass analysis was performed in the refraction mode at a resolution of about 10k. All samples were analysed using trans-2-[3-(4-tertbutylphenyl)-2methylprop-2-enylidene] malononitrile (DCTB) as a matrix. Polymer samples were dissolved in THF to obtain $1 \mathrm{mg} / \mathrm{mL}^{3}$ solution. Additionally, $50 \mu \mathrm{L}^{3}$ of 2 $\mathrm{mg} / \mathrm{mL}^{3} \mathrm{NaI}$ solution in acetonitrile were added to the polymer solution.

WAXS measurements were performed in a Bruker D8 Advance diffractometer working in parallel beam geometry. By using a Göbel mirror, the originally divergent incident X-ray beam from a line focus X-ray tube $(\mathrm{Cu})$ is transformed into $\mathrm{CuK} \alpha$ transition photons of wavelength $\lambda=1.54 \AA$. The linear detector LYNXEYE used presents an active area of $14.4 \mathrm{~mm} \times 16 \mathrm{~mm}$. The scattering angle $2 \theta$ was varied from 4 to $30^{\circ}$ with a step of $0.05^{\circ}$ in reflection geometry, measuring during $10 \mathrm{~s} \mathrm{point}^{-1}$.

SAXS was used to investigate the large-scale structural features by a Rigaku 3pinhole PSAXS-L equipment. $\mathrm{CuK}_{\alpha}$ transition photons of wavelength $\lambda=1.54 \AA$ are produced by a MicroMax-002+ X-ray generator system, which is composed by a microfocus sealed tube source module and an integrated X-ray generator unit. The scattered X-ray are detected on a two-dimensional multiwire X-ray detector (Gabriel design, 2D-200X) with about $200 \mu \mathrm{m}$ resolution and $200 \mathrm{~mm}$ diameter active area. The flight path and the sample chamber in this equipment are under vacuum. The azimuthally averaged scattered intensities were obtained as a function of wave vector $q$, $q=4 \pi \lambda^{-1} \sin \theta$. Silver behenate was used as standard for reciprocal space calibration. The experiments were carried out at $25^{\circ} \mathrm{C}$, using a Linkam scientific instruments THMS 600 temperature controller.

Perkin Elmer Pyris 1 differential scanning calorimetry (DSC) equipped with a refrigerated cooling system Intracooler $2 \mathrm{P}$, was used to investigate the thermal properties. All the experiments were performed under nitrogen flow and the instrument was calibrated with an indium standard. 
To erase any previous thermal history, the samples were melted in the DSC for 3 $\min$ at $165^{\circ} \mathrm{C}$. Then the samples were cooled at $5{ }^{\circ} \mathrm{C} / \mathrm{min}$ and heated at the same rate to register the cooling and the subsequent heating scans.

Isothermal crystallization experiments were performed by the procedure recommended by Lorenzo et al. ${ }^{43}$ In the present case, it was impossible to perform overall isothermal crystallization experiments in the DSC by directly quenching the PLA samples from the melt. However, if the sample is first cooled to the glassy state, nucleation can be greatly enhanced and then the polymer is able to crystallize upon heating from the glassy state. ${ }^{44,45}$ Therefore, for isothermal crystallization experiments from the glassy state, samples were cooled from $165^{\circ} \mathrm{C}$ to $20^{\circ} \mathrm{C}$ at $60{ }^{\circ} \mathrm{C} / \mathrm{min}$ (a temperature below the $T_{g}$ ) to induce nucleation. Finally, the samples were heated at a rate of $60^{\circ} \mathrm{C} / \mathrm{min}$ until the chosen isothermal crystallization temperature $\left(T_{c}\right)$.

Self-nucleation is a thermal technique designed to enhance nucleation density. Crystal fragments or chain segments with residual crystal memory are the best nucleating source for any polymer. The SN procedure applied here consists of several steps. $^{46-48}$

a. Erasure of previous thermal history and crystalline memory by heating the samples to $165^{\circ} \mathrm{C}$ for $3 \mathrm{~min}$. All thermally sensitive nuclei are destroyed in this step leaving only temperature-resistant heterogeneous nuclei.

b. Creation of the initial "standard" semicrystalline state by cooling the molten sample at $20^{\circ} \mathrm{C} / \mathrm{min}$ down to $20^{\circ} \mathrm{C}$. The cooling scan recorded is the "standard" one (and the peak crystallization temperature measured is the standard $T_{c}$ ), since it is a function of the number density of thermally stable nuclei of the polymer sample.

c. The sample is heated at $20^{\circ} \mathrm{C} / \mathrm{min}$ from $20^{\circ} \mathrm{C}$ up to a selected self-nucleation temperature (denoted $T_{s}$ ), and then the sample is held at this $T_{s}$ temperature for 5 min. During this period of time at $T_{s}$, the sample could melt, self-nucleate or self-nucleate and anneal depending on the $T_{s}$ value.

d. Subsequent cooling at $20^{\circ} \mathrm{C} / \mathrm{min}$ from $T_{s}$ down to $20^{\circ} \mathrm{C}$.

e. Final melting. The sample is heated from $20^{\circ} \mathrm{C}$ to above its melting point at 20 ${ }^{\circ} \mathrm{C} / \mathrm{min}$ to record the full melting behavior.

The effects of the self-nucleation treatment are reflected in the crystallization (step d) and melting of the material (step e), according to the selected $T_{s}$. 
If $T_{s}$ is very high, the sample is said to be in Domain I or complete melting domain, i.e., when complete melting occurs and the crystalline memory of the material is erased. If $T_{s}$ is high enough to melt the sample almost completely, but low enough to induce self-nucleation without causing annealing, the sample will be under Domain II or exclusive self-nucleation domain. If $T_{s}$ is sufficiently low, only part of the crystalline population will be molten and therefore the unmelted crystals will undergo annealing for 5 minutes during step (c), the sample is said to be in Domain III or self-nucleation and annealing domain.

Thermal fractionation by Successive Self-Nucleation and Annealing (SSA) experimental protocol applies a temperature program that is designed to produce distinct thermal fractions by molecular segregation during crystallization, self-nucleation and annealing, in a fast and efficient way. ${ }^{47,49,50}$

The procedure to apply SSA is shown schematically in Figure 1 and described below:

a. Erasure of thermal history by heating the sample to $165^{\circ} \mathrm{C}$ for $3 \mathrm{~min}$.

b. Creation of a "standard" thermal history by cooling to $20^{\circ} \mathrm{C}$ at $20^{\circ} \mathrm{C} / \mathrm{min}$. Then, the sample is equilibrated at $20^{\circ} \mathrm{C}$ for $1 \mathrm{~min}$.

c. The sample is heated at $20^{\circ} \mathrm{C} / \mathrm{min}$ to a partial melt temperature denoted $T_{s \text { (ideal). }}$ This $T_{s(\mathrm{ideal})}$ must be determined in separate $\mathrm{SN}$ experiments (as explained above). This temperature corresponds to the lowest $T_{s}$ value within Domain II, or the $T_{s}$ value that is capable of inducing maximum self-nucleation without annealing.

d. The sample is held at this $T_{s}$ for $5 \mathrm{~min}$. This isothermal fractionation time is kept constant in the following different SSA steps.

e. The sample is cooled at a constant rate from $T_{s(\text { ideal })}$ to $20^{\circ} \mathrm{C}$, so the polymer will crystallize during cooling after self-nucleation. Then the sample is held at $20^{\circ} \mathrm{C}$ for $1 \mathrm{~min}$.

f. The sample is heated at $20^{\circ} \mathrm{C} / \mathrm{min}$ from $20^{\circ} \mathrm{C}$ to $T_{s(1)}$. It will be held at $T_{s(1)}$ for 5 min. The difference in temperature between $T_{s(\text { ideal })}$ and $T_{s(1)}$ was set at $5{ }^{\circ} \mathrm{C}$. This is the fractionation window, since it determines the size of the thermal fraction and it should be kept constant throughout the SSA experiment.

g. The sample is again cooled down, but in this case from $T_{s(1)}$ to $20{ }^{\circ} \mathrm{C}$ at 20 ${ }^{\circ} \mathrm{C} / \mathrm{min}$ and held at $20^{\circ} \mathrm{C}$ for $1 \mathrm{~min}$. 
h. Steps "f" and "g" are repeated at progressively lower $T_{s}$ values. In this work, 9 cycles with $T_{s}$ temperatures in Domain III were employed.

i. Finally, the sample is heated at $20{ }^{\circ} \mathrm{C} / \mathrm{min}$ to the melt state, during this final heating run the consequences of SSA fractionation are revealed.

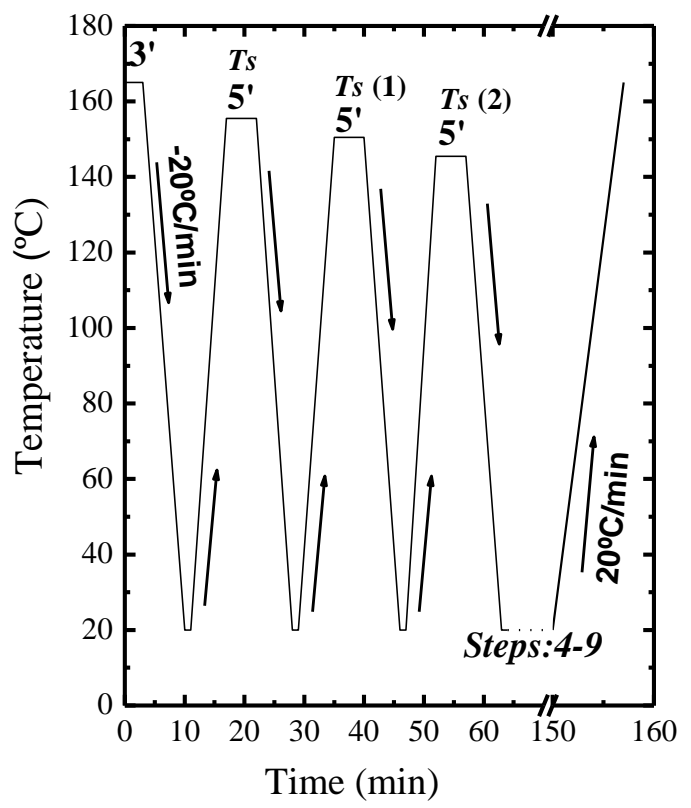

Figure 1. SSA thermal protocol schematic representation; see text for accurate description of the experimental protocol.

Spherulite growth rate experiments were performed by recording their growth in a Leitz Aristomet polarized light optical microscope, equipped with a $\lambda$ plate in between the polarizers at $45^{\circ}$. Film samples with around $20 \mu \mathrm{m}$ thickness were heated to $165^{\circ} \mathrm{C}$ for $3 \mathrm{~min}$ and then crystallized from the melt by cooling to the chosen crystallization temperature $\left(T_{c}\right)$ at $20{ }^{\circ} \mathrm{C} / \mathrm{min}$. A temperature controlled hot stage (Mettler FP82HT) was used for these experiments and the dimensions of the spherulites were periodically registered with a Leica DC420 digital camera. Additionally, the numbers of active nuclei were counted as a function of time with the same equipment.

\section{Results and discussion}

Cyclic poly(L-lactide) (c-PLLA) and poly(D-lactide) (c-PDLA) were obtained by $\mathrm{CuAAC}$ reaction on end-functionalized linear poly(L-lactide) ( $l$-PLLA) and poly(Dlactide) (l-PDLA), respectively.

The efficiency of the cyclization was determined by SEC, MALDI-MS, and quantitative ${ }^{1} \mathrm{H}$ NMR spectroscopy. In general, a cyclization reaction leads to a more 
compact coil that results in a smaller hydrodynamic volume; hence, cyclic polymers show higher retention time. SEC chromatograms on Figure 2 can be a direct and easy analysis of the cyclization efficiency. According to the above, a shift of the molar mass distribution of linear polymer $\left(\mathrm{M}_{\mathrm{n}}^{\mathrm{SEC} \text {,app }}(l\right.$-PLLA $)=16700 \mathrm{~g} / \mathrm{mol} ; \bigoplus_{\mathrm{M}} 1.10$ and $\mathrm{M}_{\mathrm{n}}^{\mathrm{SEC} \text {,app }}(l$-PDLA $\left.)=16200 \mathrm{~g} / \mathrm{mol} ; \bigoplus_{\mathrm{M}} 1.11\right)$ to higher elution volume, combined with the retention of the molar mass distribution attest the efficiency of the cyclization reaction, giving rise to cyclic polymer $\left(\mathrm{M}_{\mathrm{n}}^{\mathrm{SEC} \text {,app }}(c\right.$-PLLA $)=14000 \mathrm{~g} / \mathrm{mol}$; $\bigoplus_{\mathrm{M}} 1.21$ and $\left.\mathrm{M}_{\mathrm{n}}{ }^{\mathrm{SEC}, \text { app }}(c-\mathrm{PDLA})=13900 \mathrm{~g} / \mathrm{mol} ; \bigoplus_{\mathrm{M}} 1.22\right)$.

Figure 2 reveals the presence of shoulders in the SEC traces at low retention times. To date, except in very peculiar conditions, the preparation of pure macrocycles is always accompanied by the presence of few poisoning impurities, such as linear chains or longer cycles. Since ${ }^{1} \mathrm{H}$ NMR analyses (cfr., hereafter) do not reveal the presence of protonic end-groups, those entities are mainly constituted by interconnected chains, giant macrocycles or possible catenanes. ${ }^{41}$ Deconvolutions of both SEC chromatograms estimate a level of impurity close to $15 \mathrm{~mol} \%$.

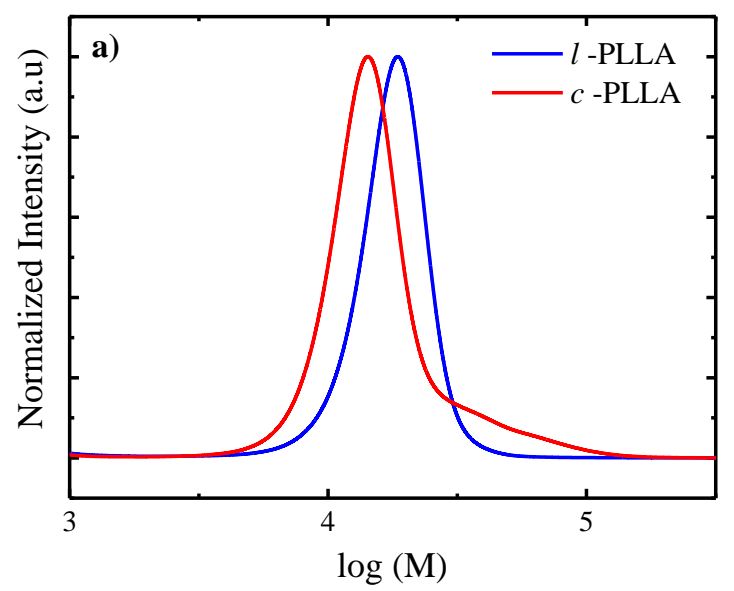




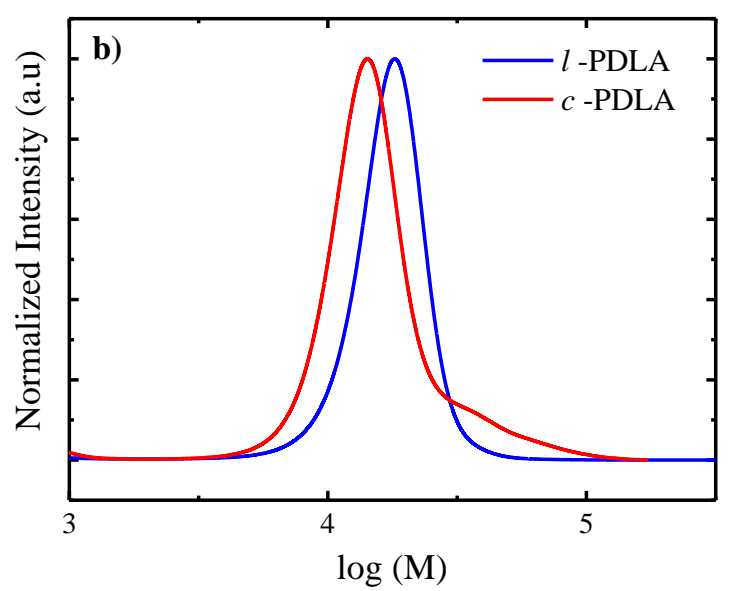

Figure 2. SEC chromatograms of the linear precursor and the cyclic polymer. a) linear and cyclic PLLA and b) linear and cyclic PDLA. (THF/NEt 3 as eluent)

Besides, Figure 3a and $\mathrm{b}$ show the MALDI-TOF MS spectra where the linear precursor and cyclic polymer reveal a common major distribution, the molecular weight of the linear and cyclic polymer samples remained unchanged after the click cyclization, so are therefore indistinguishable based on this signal only. On the contrary, the linear polymer is prone to the loss of $\mathrm{N}_{2}$ (Figure 3c), while for cyclic polymer this loss is not observed. The protonated ions of cyclic appear as a signature for the triazole group, due to its pronounced basicity (Figure 3d), as suspected following the report of Li et al. ${ }^{51}$

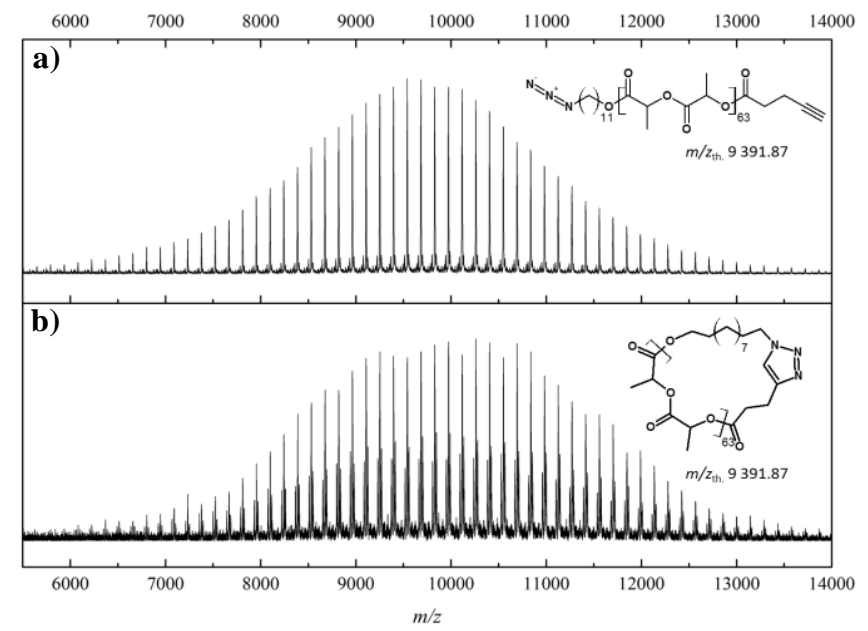




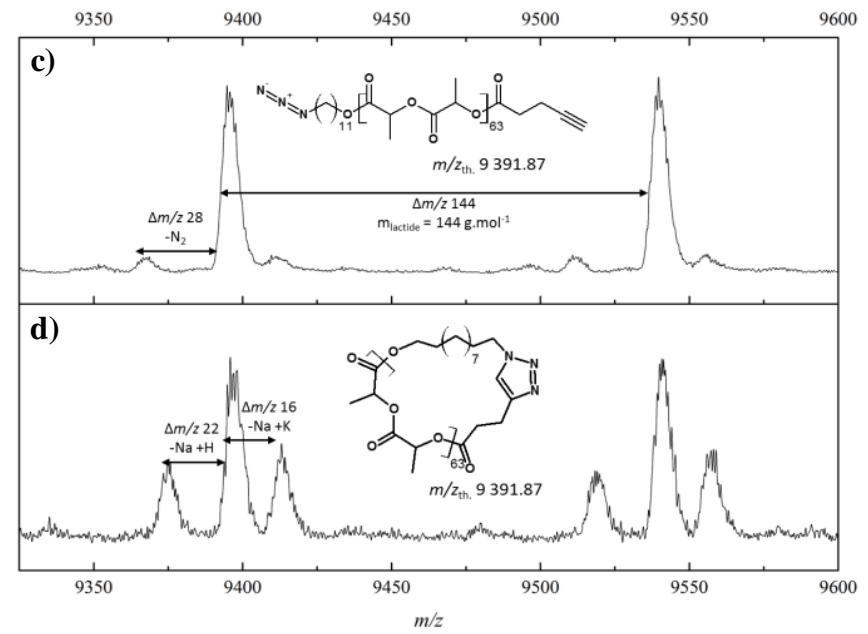

Figure 3. $a$ and b) MALDI-MS spectra of linear (above) and cyclic (below) PLLA. NaI as cationizing agent. $c$ and d) Zoom on the MALDI-MS spectra of linear (above) and cyclic (below) PLLA. NaI as cationizing agent.

The cyclization and the high conversion is also confirmed in the ${ }^{1} \mathrm{H}$ NMR spectra, by the complete disappearance of characteristic signal for linear precursor PLLA, $\delta=1.98, \delta=2.52$ and $\delta=3.25$ ppm (protons $i, h$ and $a$, respectively, in Figure $4 \mathrm{a}$ and also the presence of the characteristic signals $\delta=3.06, \delta=4.28$ and $\delta=7.36$ ppm (protons $a, h$ and $i$, respectively, in Figure 4b) of the macrocyclic $c$-PLLA.

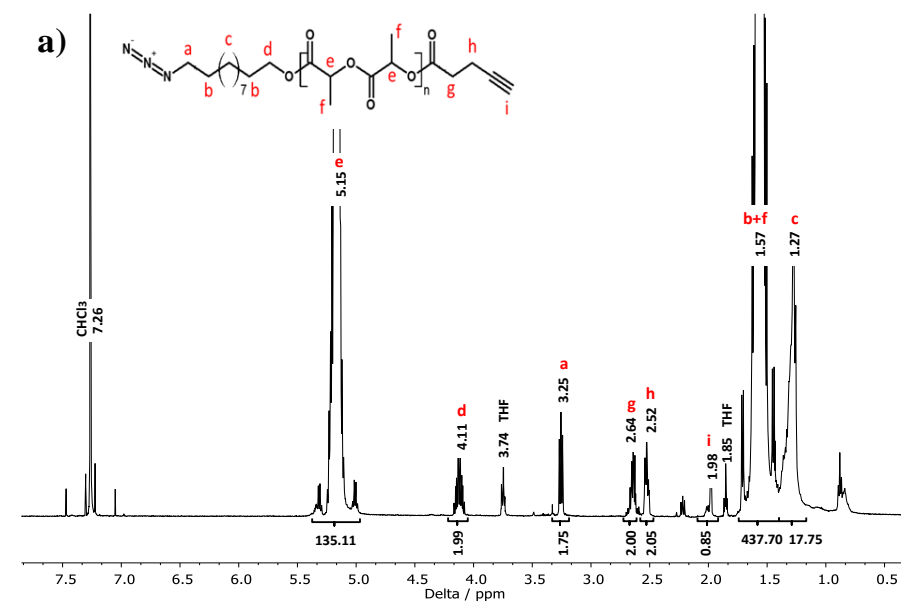




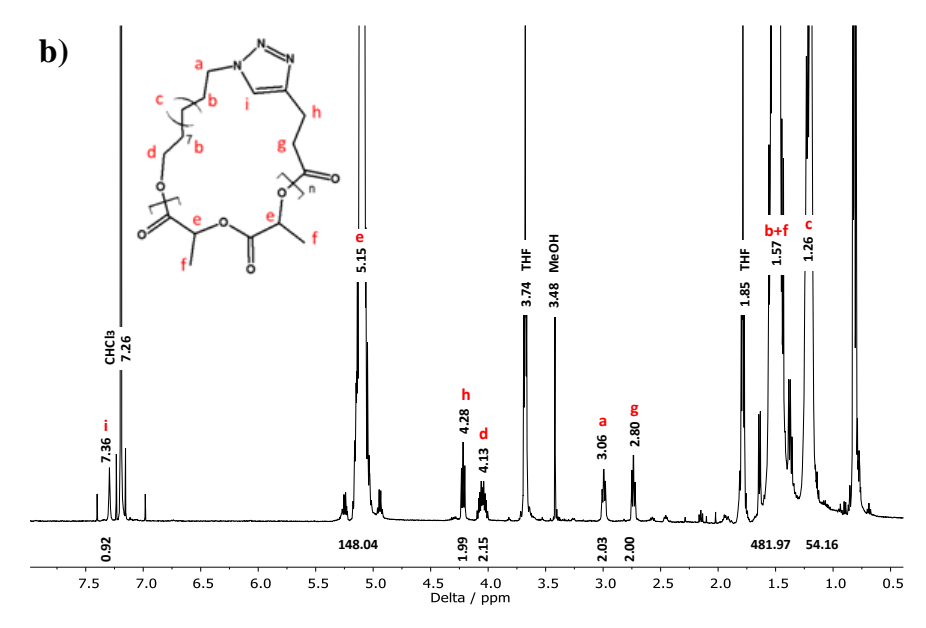

Figure 4. ${ }^{1} \mathrm{H}$ NMR spectra of a) linear PLLA and b) cyclic PLLA ( $\left.\mathrm{CDCl}_{3}, 500 \mathrm{MHz}\right)$.

\section{Non-isothermal DSC Experiments}

Figure 5 shows standard crystallization and melting behavior of linear and cyclic PLLA and PDLA with $M_{n}$ value of $10 \mathrm{~kg} / \mathrm{mol}$. The cooling scan was measured after the crystalline thermal history of the material was erased. Then the subsequent heating scans recorded the cold-crystallization and melting of the crystals during heating at 5 ${ }^{\circ} \mathrm{C} / \mathrm{min}$. The relevant parameters obtained from these DSC experiment are listed in Table 1.

Neither linear PLLA and PDLA nor cyclic PLLA and PDLA polymers are able to crystallize during cooling from the melt (see Figure 5a) at the scanning rate employed ( $\left.5^{\circ} \mathrm{C} / \mathrm{min}\right)$. Only the vitrification process at the $T_{g}$ can be observed. In the heating scan, the glass transition is clearly observed, followed by a prominent cold-crystallization exotherm and finally a complex melting behaviour, where bimodal endotherms are observed (see Figure 5b).

Table 1 shows that in all cases cyclic samples have lower cold crystallization temperatures $\left(T_{c c}\right)$ than their linear analogues. This indicates a higher nucleating capacity of the cyclic PLAs as compared to the linear chains. This effect is possibly due to the more compact coil conformation of the cyclic chains and to the larger supercooling of cyclic molecules. ${ }^{2}$ These aspects will be discussed in detail below.

Regarding the experimentally determined, apparent peak melting temperatures $\left(T_{m}\right)$ of the cyclic polymer, they are also slightly higher (i.e., $2^{\circ} \mathrm{C}$ ) than those of the linear PLLAs or PDLAs. The difference is not very large in this case, but it is 
qualitatively similar to that found in the case of polycaprolactones by Müller et al. ${ }^{28,27}$ Therefore, according to their thermal behavior, these PLA systems of different chain topologies (linear vs cyclic) would fall within group 3 described in the introduction.

On the other hand, the melting endotherms are bimodal, with two melting points, as a consequence of partial fusion and recrystallization during the heating scan and not to the presence of two crystalline forms (see WAXS studies below, the results corroborate the existence of a single crystalline form (i.e., $\alpha$ form)). ${ }^{52,53}$
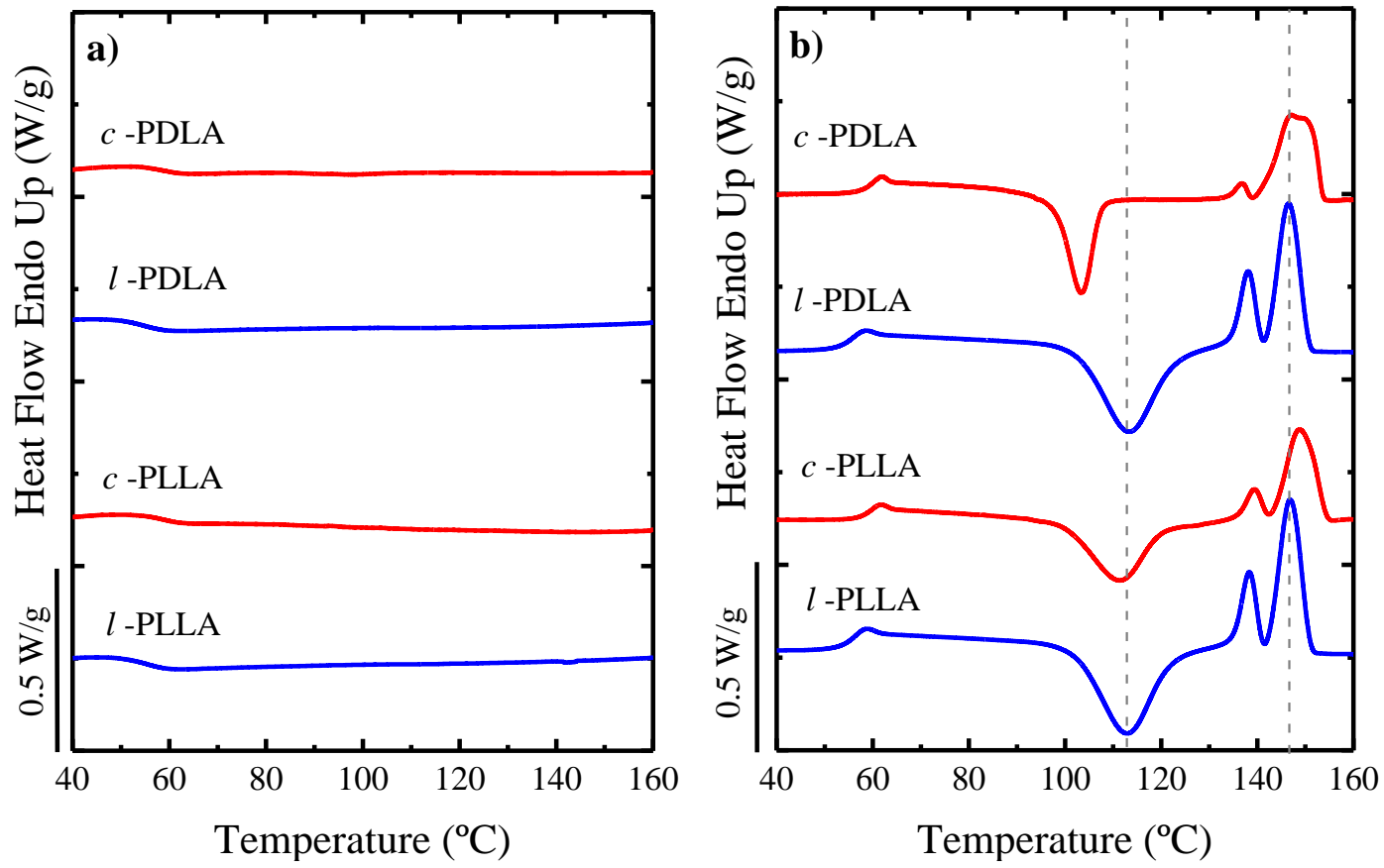

Figure 5. a) Cooling DSC scans from the melt and b) subsequent heating scans for the indicated samples. Scanning rate $5{ }^{\circ} \mathrm{C} / \mathrm{min}$.

PLAs cannot crystallize during cooling at $5{ }^{\circ} \mathrm{C} / \mathrm{min}$, confirming that the PLAs employed here exhibit a slow overall non-isothermal crystallization rate when cooled from the melt. However, when they are subsequently heated from the glassy state, they undergo cold-crystallization followed by melting. The values of cold crystallization and melting enthalpies (see Table 1) are nearly identical within the error of the measurements (typically 5-10\%), indicating that all crystallization takes place during heating. Additionally, the amount of crystallinity that the samples develop during the heating run has been calculated and reported in Table 1 . All crystallinity values for 
linear and cyclic PLAs are very similar taking into account the typical errors involved in these estimations (i.e., 10\%).

Finally, the glass transition temperature $\left(T_{g}\right)$ was around $54{ }^{\circ} \mathrm{C}$ and $58{ }^{\circ} \mathrm{C}$ for linear and cyclic PLA, respectively. So the cyclic polymers exhibit higher glass transition temperatures than their linear counterparts, in view of their lack of highly mobile chain ends, which are present in their linear counterparts. Similar increases in glass transition values in comparison to linear analogue chains have been reported for other cyclic polymers. ${ }^{13}$

Table 1. Thermal transitions determined by DSC (at $5{ }^{\circ} \mathrm{C} / \mathrm{min}$ ) of cyclic and linear materials.

\begin{tabular}{ccccccc}
\hline & $\mathbf{T}_{\mathbf{g} \text { onset }}\left({ }^{\circ} \mathbf{C}\right)$ & $\mathbf{T}_{\mathrm{cc}}\left({ }^{\mathbf{O}} \mathbf{C}\right)$ & $\Delta \mathbf{H}_{\mathrm{cc1}}(\mathbf{J} / \mathbf{g})$ & $\mathbf{T}_{\mathbf{m}}\left({ }^{\mathbf{O}} \mathbf{C}\right)$ & $\Delta \mathbf{H}_{\mathbf{m}}(\mathbf{J} / \mathbf{g})$ & ${ }^{a} \mathbf{X}_{\mathrm{c}}(\%)$ \\
\hline $\boldsymbol{l}$-PLLA & 54 & 113.0 & -36.0 & $138.5 / 146.8$ & 37.1 & 61 \\
$\boldsymbol{c}$-PLLA & 58 & 111.2 & -23.8 & $139.5 / 148.6$ & 24.3 & 55 \\
$\boldsymbol{l}$-PDLA & 53 & 113.3 & -34.5 & $138.2 / 146.5$ & 36.9 & 61 \\
$\boldsymbol{c}$-PDLA & 58 & 103.4 & -20.2 & $136.8 / 148.5$ & 26.1 & 59 \\
\hline
\end{tabular}

${ }^{a}$ Estimation of the degree of crystallinity developed by PLA during the heating run based on the melting enthalpy, employing $\Delta H_{m}{ }^{\circ} \sim 60 \mathrm{~J} / \mathrm{g}$ for linear and $\Delta H_{m}{ }^{\circ} \sim 44 \mathrm{~J} / \mathrm{g}$ for cyclic PLAs, as reported by Sugai et al. ${ }^{31}$

\section{Crystal structure}

As demonstrated above (Figure 5), if a cooling rate of $5^{\circ} \mathrm{C} / \mathrm{min}$ is employed, the samples are unable to crystallize during cooling from the melt, but they can do it during heating (also at $5{ }^{\circ} \mathrm{C} / \mathrm{min}$ ) from the glassy state. In order to perform the WAXS experiments, the samples were first cooled from the melt to room temperature at 20 ${ }^{\circ} \mathrm{C} / \mathrm{min}$ (and no crystallization took place) and then heated to $110{ }^{\circ} \mathrm{C}$ at $20{ }^{\circ} \mathrm{C} / \mathrm{min}$. At $110{ }^{\circ} \mathrm{C}$, the WAXS measurements were performed isothermally, as taking the WAXS spectra in the $2 \theta$ range employed and with the resolution adopted took $60 \mathrm{~min}$.

In Figure 6, the WAXS diffractograms of linear and their analogous cyclic PLLA and PDLA polymers are shown. The dominant diffraction peaks in linear and cyclic samples are observed at $2 \theta$ values of $14.4^{\circ}, 16.4^{\circ}, 18.7^{\circ}$ and $22.0^{\circ}$ that can be attributed to reflections from (010), (110) and/or (200), (203) and (115) planes respectively. The WAXS patterns can therefore be assigned to the $\alpha$ form of PLA, which is orthorhombic with parameters $\mathrm{a}=10.7 \AA, \mathrm{b}=6.45 \AA$ and $\mathrm{c}$ (fiber axis) $=27.8$ $\AA$, where the molecules are assumed to have a left-handed $10_{3}$ helical conformation. ${ }^{26,31,54}$ 
Besides the strongest diffraction peaks, some weak diffraction peaks at $2 \theta=$ $13.3^{\circ}, 20.4^{\circ}, 23.7^{\circ}$ y $24.8^{\circ}$ indicative of (004/103), (204), (016) y (206) reflections are also observed in Figure 6. All these reflections represent stable $\alpha$-crystals, whereas the characteristic diffraction of PLA $\alpha^{\prime}$ - crystals at $18.4^{\circ}$ and $24.4^{\circ}$ could not be observed. ${ }^{55}$ This implies that both linear and cyclic PLA chains are able to crystallize with an identical unit cell.

In summary, the WAXS results shows that cyclic topology has no influence on the crystalline structure at the nanometric level. Similar results have been found for cyclic PCLs. ${ }^{2,27}$
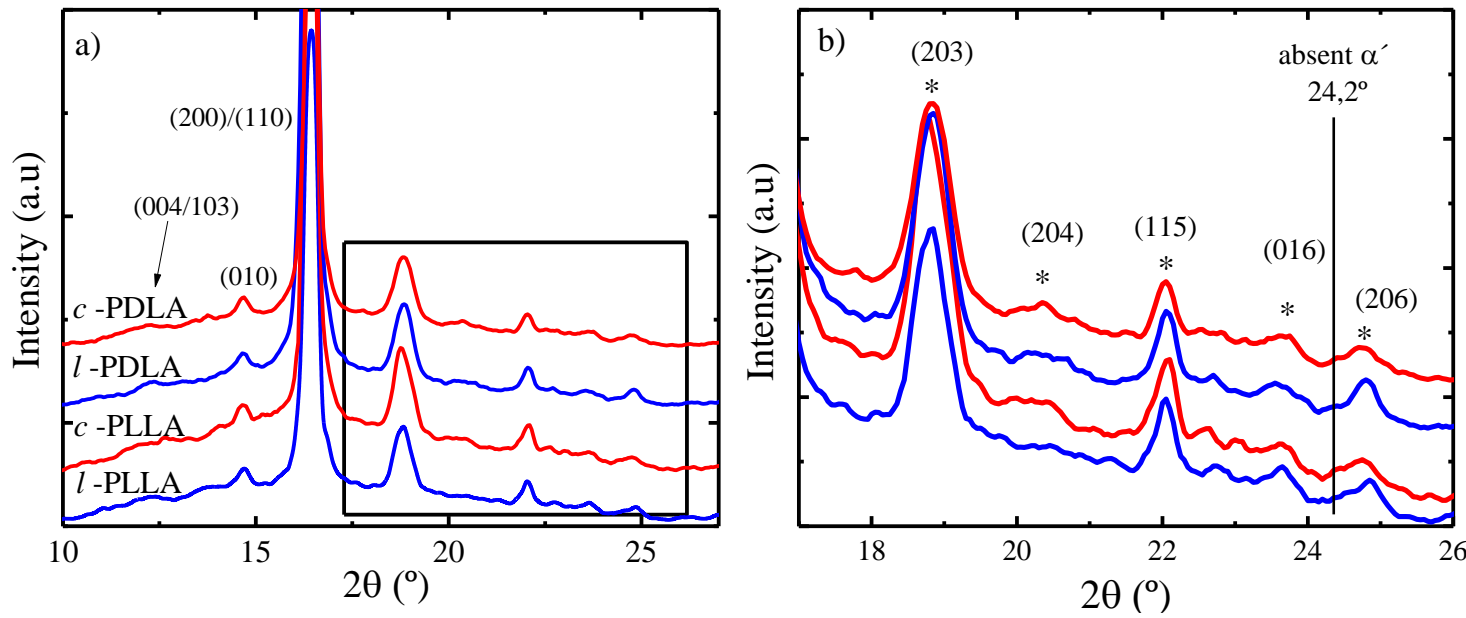

Figure 6. a) WAXS diffraction patterns for samples heated from the glassy state at $20^{\circ} \mathrm{C} / \mathrm{min}$. Measurements performed at $110^{\circ} \mathrm{C}$. b) magnification of the range $17^{\circ}$ to $26^{\circ}$ for all samples.

\section{Nucleation}

Nucleation experiments performed by polarized light optical microscopy yield values of nucleation density as a function of time. Such nucleation kinetic measurements were performed for the four samples analysed here at different temperatures, under isothermal conditions. The data for all samples is reported in Figure S1-1 in the Supporting Information.

A representative set of nucleation kinetics data can be seen in Figure 7a. Cyclic PLA chains nucleate faster than linear chains at same $T_{c}$ temperature $\left(110^{\circ} \mathrm{C}\right)$. On the other hand, if the nucleation density is compared at saturation values (long times) at a $T_{c}$ temperature of $110^{\circ} \mathrm{C}$, it is $40 \%$ higher for $c$-PLLA as compared to $l$-PLLA. Moreover, this value is $70 \%$ higher if the $c$-PDLA and $l$-PDLA are compared (see Figure S1-2 in the Supporting Information). Such improved nucleation is also confirmed by the PLOM micrographs of cyclic and linear PLLA shown in Figure $7 \mathrm{~b}$. 
The nucleation rate $(I)$ provided in Figure $7 \mathrm{~b}$ as a function of temperature was determined from the slope of the plots nucleation density as a function of time at short nucleation times, where a linear relationship could be fitted (see Figure S1-1). The observed values of nucleation rate are much higher for cyclic PLA at lower temperatures, a clear sign of a more instantaneous nucleation process as compared to linear chains. As the nucleation density decreases with $T_{c}$, at very high $T_{c}$ values, the differences in nucleation rate between cyclic and linear chains tend to disappear.

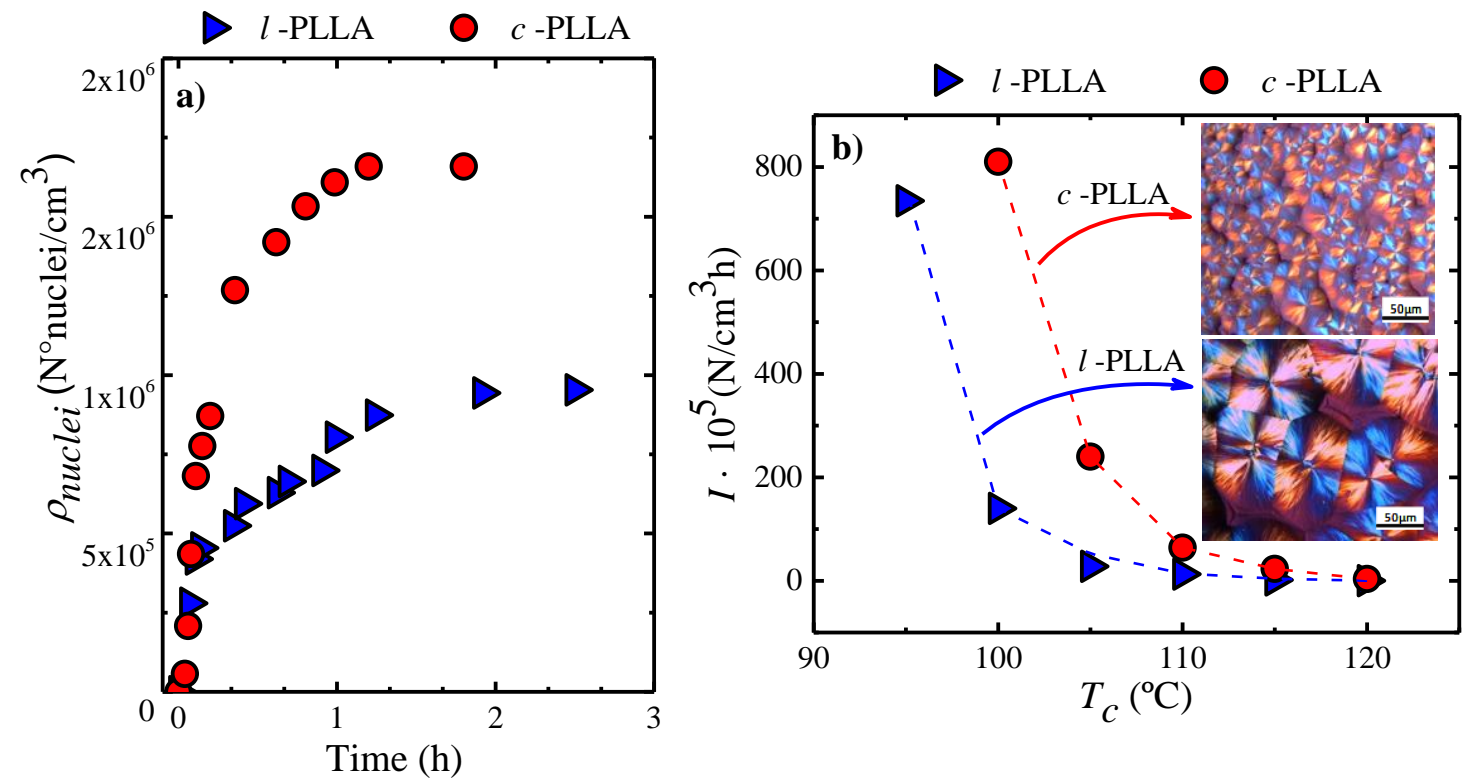

Figure 7. a) Nucleation density as a function of time for linear and cyclic PLLA at $110^{\circ} \mathrm{C}$. b) Nucleation rate (I) as a function of the crystallization temperature $\left(T_{c}\right)$.

We have focused so far on the general differences in nucleation between linear and cyclic chains. However, we also detected some differences in nucleation between $l$ PLLA and $l$-PDLA and between $c$-PLLA and $c$-PDLA. The reason behind these differences is unknown. Bulk polymers always nucleate heterogeneously. The heterogeneities that cause nucleation are catalytic debris or impurities present in the monomers and solvents employed during the synthesis. For $c$-PLLA and $c$-PDLA samples, we would expect similar amount of residual catalyst. In addition, the monomers used are analytical grade but could contain different amounts of impurities (in the ppm range). Additionally, differences in nucleation density between $c$-PLLA and $c$-PDLA could be connected with small differences in PLA "impurities" content (i.e., linear PLA chains and/or larger cyclic molecules detected by SEC in Figure 2). The 
differences in nucleation density between $l$-PLLA and $l$-PDLA should only be connected with monomer impurities, as their synthetic procedure is identical.

In summary, cyclic PLAs exhibited higher nucleation densities and higher nucleation rates than their linear analogues, especially at high supercoolings. Ring chain topologies are characterized by their more collapsed conformation in the melt and their lack of chain ends, ${ }^{56,57}$ so they are expected to nucleate easier than linear chains. Similar results have also been reported for $\mathrm{PCL},{ }^{28} \mathrm{PTHF}^{20,32}$ and $\mathrm{PE}^{24}$

\section{Spherulitic growth kinetics of cyclic and linear PLA isomers}

The spherulitic growth rate from the melt is represented as a function of the isothermal crystallization temperature $\left(100-135^{\circ} \mathrm{C}\right)$ in Figure 8 . The spherulitic growth rate $G(\mu \mathrm{m} / \mathrm{min})$ was determined from the slope of the straight line obtained when the spherulitic radius $(\mu \mathrm{m})$ is plotted as a function of time ( $\mathrm{min})$.

The morphology of the spherulites was independent of chain topology or stereochemistry for all samples examined in this work. Typical PLA negative spherulites characteristic of the alpha form were observed during isothermal crystallization (see Figure $7 \mathrm{~b}$ as an example).

For all samples, the spherulitic radius versus time plots exhibited highly linear trends over the entire range of investigated $T_{c}$, implying that the growth rate was independent of the size of the spherulites. The curves of spherulitic growth rate versus $T_{c}$ (Figure 8) display the typical bell-shape trend caused by the competition between thermodynamic control of secondary nucleation and diffusion.

The results of Figure 8 indicate that cyclic and linear PLAs exhibit similar spherulitic growth for both types of PLA samples. In fact, l-PLAs display slightly higher growth rates than $c$-PLAs, however, the differences are not very significant. These results are not expected, and they are not consistent with those obtained for PCLs, where the spherulites of cyclic polymers have been shown to grow faster than those of their linear analogues. ${ }^{21,28}$ As cyclic molecules have a faster diffusion rate than linear ones, it is expected that spherulitic growth rates should be larger for cyclic chains. $^{2}$

The similarities in spherulitic growth between cyclic and linear samples may be attributed to the presence of impurities in the cyclic samples, constituted either by linear chains or by cyclic molecules of higher molecular weight. López et al. ${ }^{36}$ have recently 
demonstrated that growth rates of cyclic PCL chains can be significantly decreased by the presence of small amounts of linear chains, as they thread though the cyclic chains and hinder diffusion.

In the present case, Figure 2 shows that there is a difference in the chromatograms of the linear and cyclic chains, as the cyclic chains exhibit a shoulder at low elution times (or higher molecular weights). As in our case, NMR results do not evidence the presence of protonic end-groups, the anomalous high molecular weight small fraction present in the cyclic PLAs is probably due to intermolecular reactions that yield longer cyclic molecules or interconnected linear chains. Such longer rings or linear chains, may also have a similar threading effects on the smaller (and dominant fraction) cyclic chains hindering their diffusion. In this way, such "impurities" cause a decrease in the spherulitic growth rate that makes the growth kinetics of cyclic PLAs similar to that of linear PLAs.
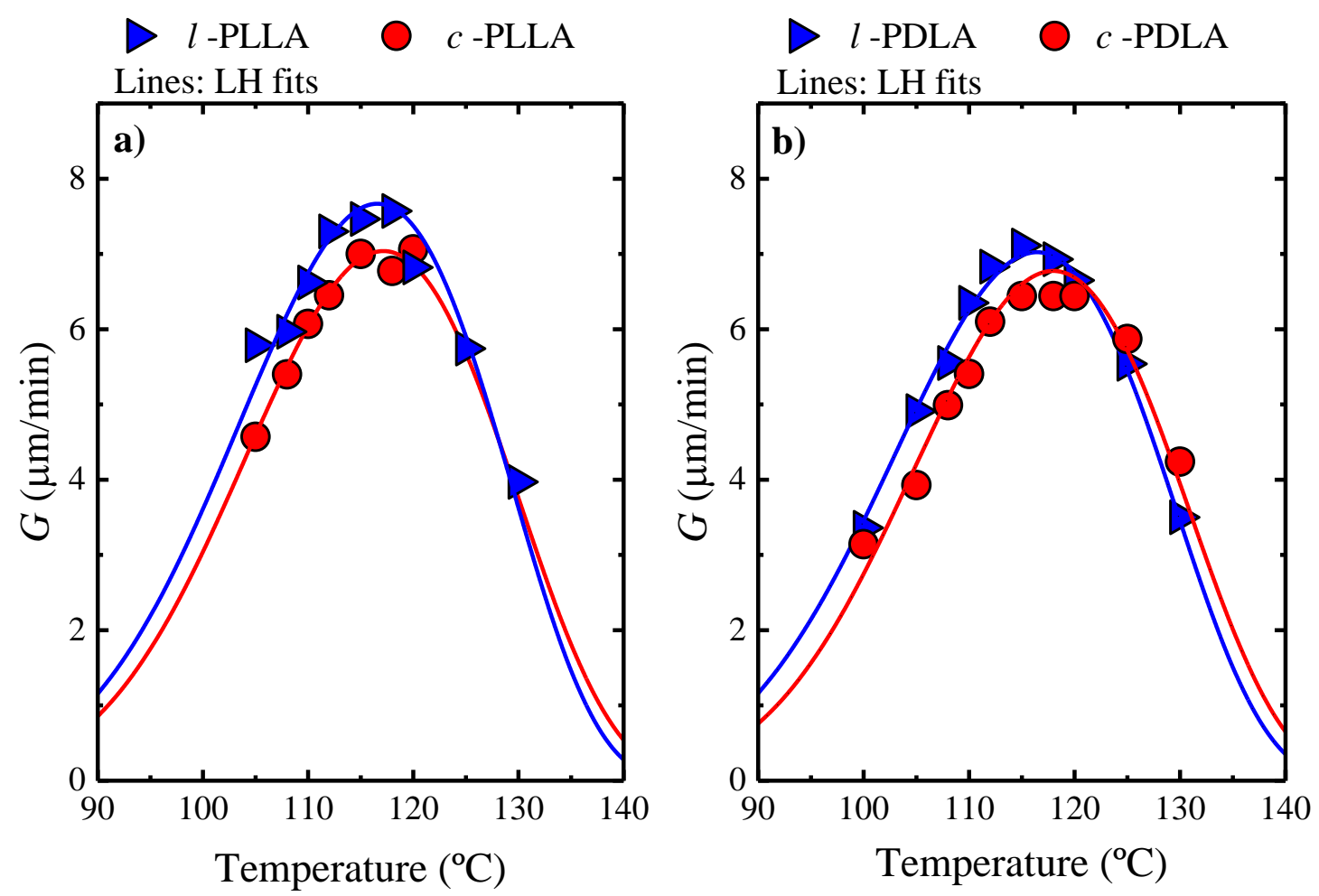

Figure 8. Spherulitic growth determined by PLOM for the indicated cyclic and linear PLA samples. The solid lines are the fits to the Lauritzen-Hoffman $(L H)$ theory (details of how the fit was performed can be found in the Supporting information, S2)

\section{Overall Isothermal Crystallization}

In this paper, isothermal crystallization experiments were performed by DSC to determine the overall crystallization rate of PLAs with linear and cyclic topology. The 
inverse of the half crystallization time provides an experimental measure of the overall crystallization rate, which includes both nucleation and growth. The overall isothermal crystallization experiments were performed by previously cooling the samples (which cannot crystallize during cooling from the melt at $60^{\circ} \mathrm{C} / \mathrm{min}$ ) to the glassy state in order to enhance their nucleation. Then, the samples were heated from $25{ }^{\circ} \mathrm{C}$ to $T_{c}$ at a rate of $60{ }^{\circ} \mathrm{C} / \mathrm{min}$ (to avoid crystallization during heating) and the isothermal crystallization was recorded in the calorimeter as a function of time.

Figure 9a and Figure 9b show the experimental overall crystallization rate $\left(1 / \tau_{50 \%}\right)$ from the glassy state as a function of $T_{c}$ for linear PLLA and PDLA and their analogous cyclic polymers. All the samples display the typical bell-shape trend, where the crystallization rate goes through a maximum as the kinetics changes from nucleation control at higher temperatures to diffusion control at lower temperatures.

The differences in crystallization kinetics from the glassy state between cyclic and linear PLA are striking. Figure 9 reports that cyclic chains require lower supercooling or higher $T_{c}$ values to crystallize than linear chains. In addition, the overall crystallization rate is much faster for cyclic chains than for analogous linear chains regarless of the stereochemistry involved in L or D samples. For example, comparing the samples with D-stereoisomers, at a constant $T_{c}$ value of $115{ }^{\circ} \mathrm{C}$, cyclic PDLA display $40 \%$ faster crystallization rate as compared to its linear analogue. 

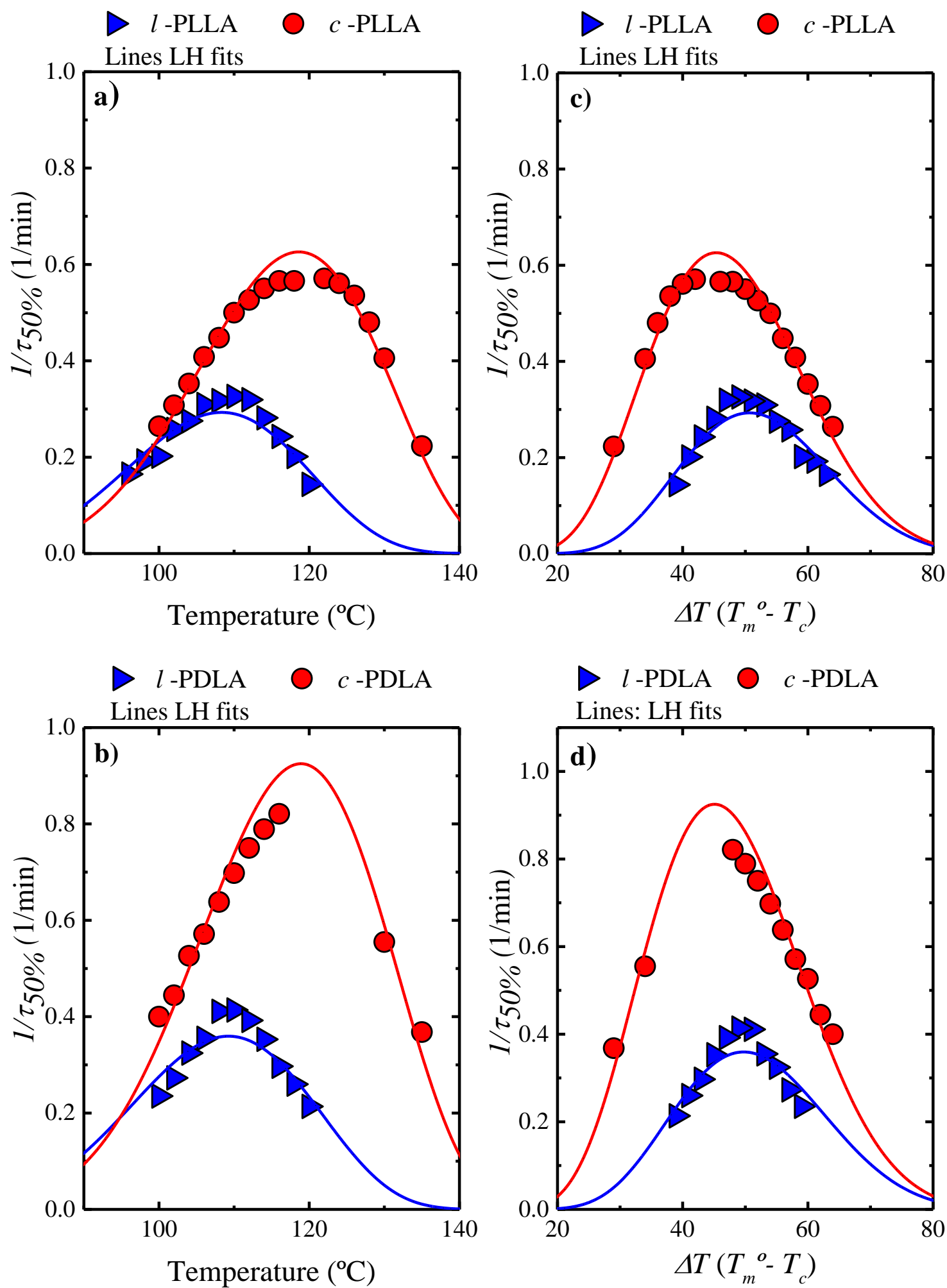

Figure 9. The inverse of half-crystallization time as a function of $T_{c}$ (a and $b$ ) and supercooling $(\Delta T)(c$ and d) for indicated PLA samples.

Additionally, Figure 9c and Figure 9d represent the overall crystallization rates, but as a function of the supercooling $\left(\Delta T=T_{m}^{0}-T_{c}\right)$, using the equilibrium melting 
temperatures $\left(T_{m}^{0}\right)$ values determined below. It can be observed that the representation as a function of supercooling shifts the curves in the $\mathrm{x}$ axis normalizing the differences in crystallization temperature exhibited by the cyclic and the corresponding linear chains. However, the curves are not superimposed on the vertical axis, as the kinetic differences between cyclic and linear chains cannot be normalized by a thermodynamic variable like $T_{m}^{0}$. These results indicate that both thermodynamic and kinetic factors are responsible for the differences observed.

In the present work, the behaviour of observed in Figure 9 can be explained taking into consideration our nucleation and growth rate results. It was shown that cyclic PLAs chains nucleate at a faster rate than linear PLAs, whereas the growth process was similar between $c$-PLA and $l$-PLA. Taking into account, that the overall crystallization process involves these two stages, the observed differences in $1 / \tau_{50 \%}$ are due to the increase in nucleation. Furthermore, as the crystallization was conducted by heating from the glassy state, the previous cooling from the melt to the glassy state also contributed to increase nucleation density. There is no doubt that the cyclic chains exhibit a higher nucleation density and a higher nucleation rate than their linear analogues, irrespective of the D or L chain configuration.

Finally, the continuous lines are fits to the Lauritzen Hoffman theory, ${ }^{58,59}$ which are discussed in the Supporting Information S2.

\section{Fitting of DSC isothermal data to the Avrami model}

The Avrami equation can describe the primary crystallization process in polymers. ${ }^{60,61}$ The equation can be written in the following form, as postulated ${ }^{43}$

$$
1-V_{c}\left(t-t_{0}\right)=\exp \left(-k\left(t-t_{0}\right)^{n}\right)
$$

where $V_{c}(t)$ is the relative volumetric transformed fraction as a function of time, $t$ is the experimental time, $t_{0}$ is the induction time for crystallization, $n$ is the Avrami index and $k$ is the overall crystallization rate constant. The fits to the Avrami equation were performed using the Origin plug-in developed by Lorenzo et al. ${ }^{43}$ and Figure 10 shows a representative fit of Avrami model for the crystallization of $c$-PDLA at $100{ }^{\circ} \mathrm{C}$. 

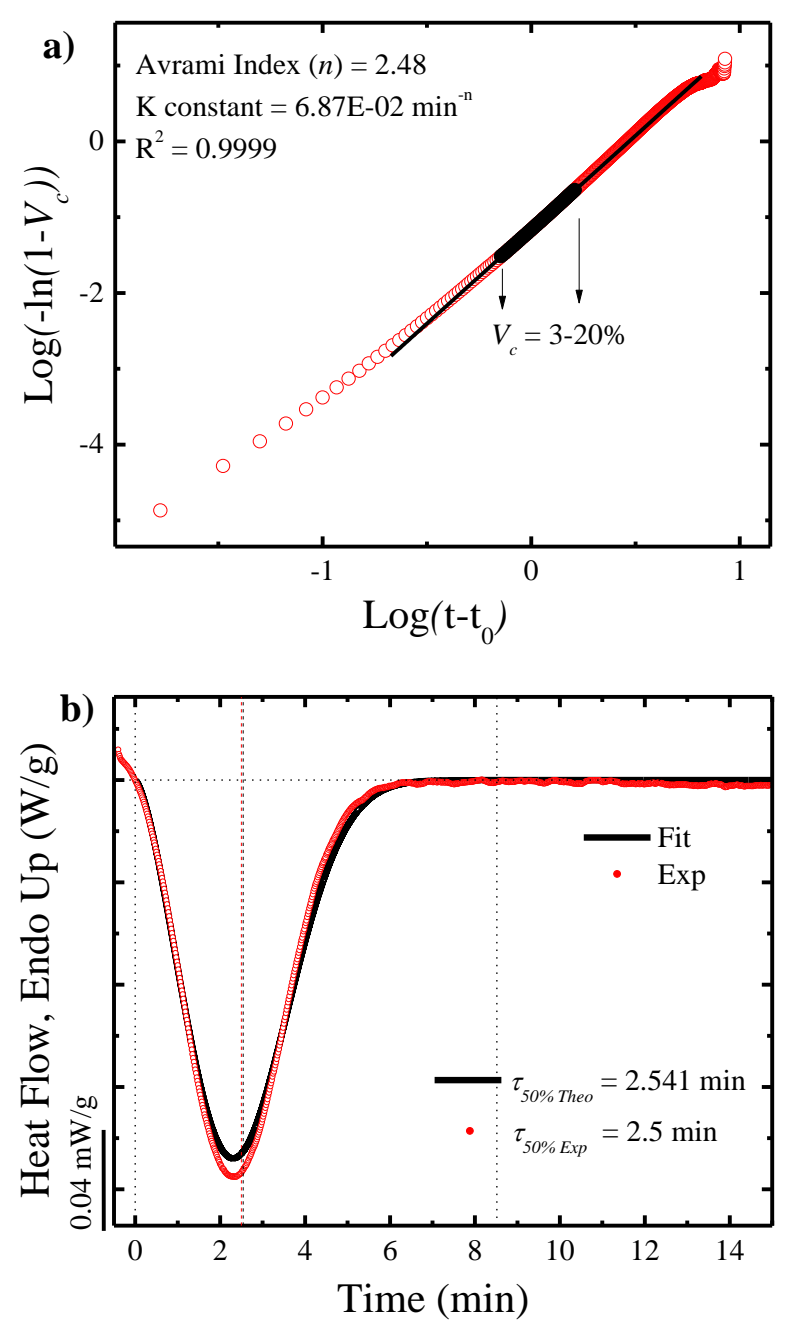

Figure 10. Isothermal crystallization studied by DSC. a) Avrami plot of the data obtained during crystallisation of cyclic PDLA at $100^{\circ} \mathrm{C}$. Raw heat flow data of c-PDLA during crystallization at $100^{\circ} \mathrm{C}$, compared to the data predicted by the Avrami model.

Figure 10a shows the double logarithmic representation that linearizes eqn (1), in the conversion range of $3-20 \%$ corresponding to the primary crystallization. The Avrami index, $n$, obtained as well as the $k$ value and the correlation coefficient for the fit, $R^{2}$, are included for one example, the rest of the results obtained are summarized in Supporting Information (Table S3). Using these values in eqn (1) and by differentiating the equation, the experimental heat flow data can be modeled by the Avrami equation, and it is shown in Figure 10b. 


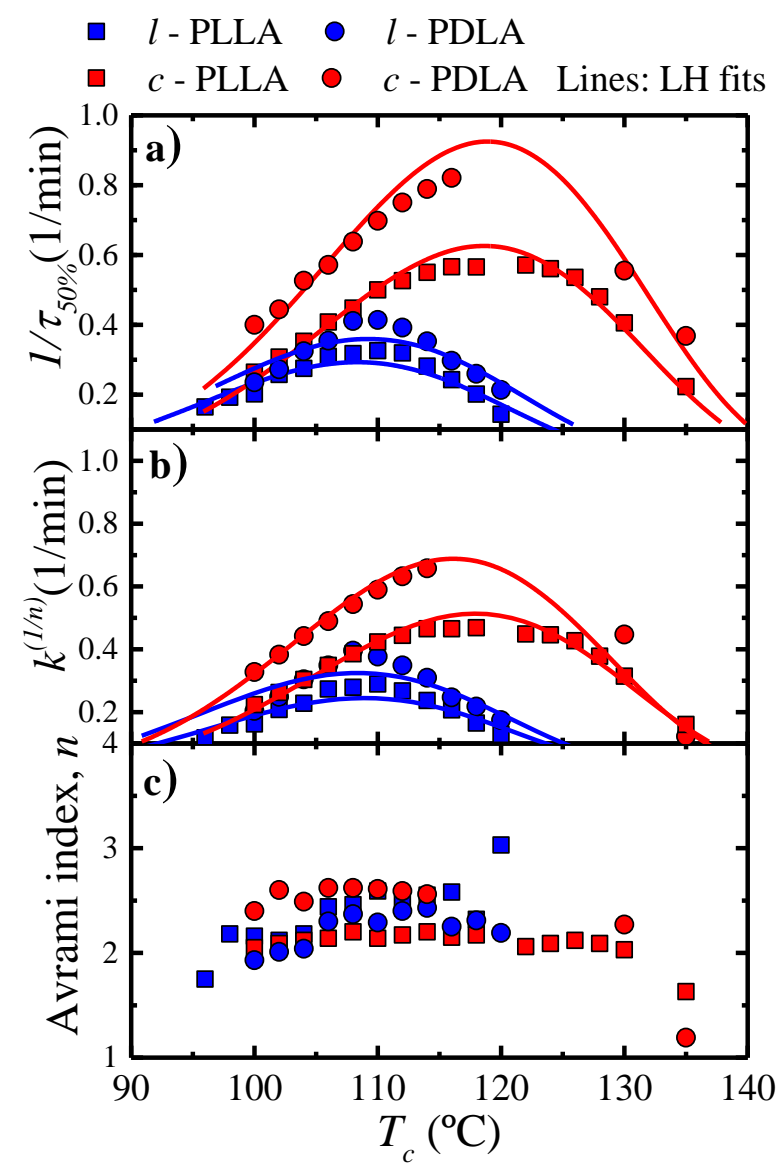

Figure 11. a) Inverse of half crystallization times for the linear and cyclic PLA as a function of $T_{c} . b$ ) Normalized rate crystallisation constant $\left(k^{1 / n}\right)$ of the Avrami model as a function of $T_{c}$. c) Avrami index as a function of $T_{c}$. Solid lines correspond to fittings of the Lauritzen-Hoffman model (see Supporting information for more details on the application of the LH model).

Figure 11 shows the representation of several kinetic parameters of crystallization as a function of the crystallization temperature. First, in Figure 11a, the inverse of the half crystallization experimental data is plotted with the LH fitting as continuous lines. The isothermal rate constant $k$ has units that depend on $n$ (i.e., $\min ^{-n}$ ), and since $n$ values are not constant, a direct comparison can not be made. One way to overcome this difficulty is to elevate $k$ to $1 / n$, so that all values of $k$ are expressed in reciprocal time units (in this case $\min ^{-1}$ ), as has been plotted in Figure $11 \mathrm{~b}$. The values of,$k^{1 / n}$ are proportional to the overall crystallization rate and, therefore, can also be adjusted to the Lauritzen and Hoffman theory, represented by the solid lines in Figure 11b. This is an excellent way to compare experimental data points (experimental values of $1 / \tau_{50 \%}$ in Figure 11a) with the Avrami theory parameters (calculated $k^{1 / n}$ values, plotted as data points in Figure 11b). The remarkable qualitative similarity between 
Figure 11a and Figure 11b is a consequence of the excellent fit of the Avrami equation to the overall crystallization rate data.

In this work, small differences are observed between $l$-PLLA and $l$-PDLA in terms of overall crystallization kinetics, as shown in Figure 11. Figure 8 demonstrates that differences in growth rate and nucleation densities are not significant. Therefore, the small differences observed in the overall crystallization kinetics in Figure 11 between $l$-PLLA and $l$-PDLA can be attributed to the higher nucleation rate observed for $l$-PDLA, which are reported in Figure S1-2 (see Supporting Information). These differences are magnified when cyclic PLAs are considered in Figure 11 and are also a consequence of the larger nucleation density and nucleation rate of cyclic PDLA as compared to cyclic PLLA. The reason why PDLA nucleates more than PLLA in cyclic materials is unknown but the possible reasons for this behavior have been discussed above (see Nucleation section).

Figure 11c shows the values of the Avrami index as a function of $T_{c}$. The values fluctuate between 2 and 3 . It must be remembered that the overall isothermal crystallization kinetics was determined by heating from the glassy state. Hence the nucleation density was greatly enhanced. Under such circumstances, when PLOM observations are made, a granular texture is observed (results not shown) which can correspond either to instantaneously nucleated spherulites $(n=3)$ or instantaneously nucleated axialites $(n=2)$. The results are therefore expected on the basis of the observed morphology. No specific trends are observed regarding stereoisomerism, chain topology or crystallization temperature.

\section{Equilibrium melting Temperature $\left(\boldsymbol{T}_{m}^{\mathbf{0}}\right)$}

In previous sections, the effect of chain topology has been found to have a very important influence on nucleation and crystallization kinetics, as expected from the faster diffusion of cyclic rings in comparison with linear analogue chains. However, it is important to ascertain if some of the differences observed can also be due to thermodynamic factors, such as the degree of supercooling applied to the material for nucleation and crystallization. This is the reason why we determine the equilibrium melting point differences between linear and cyclic PLAs. As far as the authors are aware, the equilibrium melting point of cyclic PLA chains has never been reported in the literature. 
The equilibrium melting temperature, $T_{m}^{0}$, is the melting point of a perfect crystal without surface, equivalent to a crystal in which all the chains are extended (i.e., the folded surfaces do not exist). ${ }^{59}$ It is a very important property in crystallizing polymers and not only reflects the molecular and conformational characteristic on the chain, it is also a key parameter in the analysis of crystallization kinetics. For this reason, the $T_{m}^{0}$ value will be studied for the samples $l$-PLLA, $l$-PDLA, $c$-PLLA, $c$-PDLA.

The Thompson-Gibbs approach is one of the simplest and most widely used methods to evaluate $T_{m}^{0}$, and it is based on equation $2^{61}$ :

$$
T_{m}=T_{m}^{\circ}\left(1-\frac{2 \sigma_{e}}{l \Delta h_{f}^{0}}\right)
$$

where $T_{m}^{0}$ is the equilibrium melting point, $\Delta h_{f}^{0}$ is the enthalpy per unit volume of a perfect crystal (100\% crystalline) and $\sigma_{e}$ is the fold surface free energy.

Su et al. ${ }^{27}$ derived a modified Thompson-Gibbs equation (3) for cyclic polymers by considering that the entropy of a cyclic and a linear chain in the melt are not equivalent. The modified Thompson-Gibbs equation can be written as:

$$
T_{m}=\frac{T_{m L}^{0}}{\left(1+\frac{T_{m L}^{0} \Delta S_{c y c}}{\Delta h_{f}^{0}}\right)}\left[1-\frac{2 \sigma_{e}}{\Delta h_{f}^{0} l}\right]
$$

where $T_{m L}^{0}$ is the equilibrium melting point of the linear polymer and $\Delta S_{c y c}$ is the cyclization entropy difference between the crystalline state and the melt state.

For both Thompson-Gibbs and Thompson-Gibbs modified equation, $T_{m}^{0}$ for linear and cyclic chains can be obtained by extrapolation to infinite lamellar thickness (i.e., $1 / l=0$ ), which can represent the equilibrium melting temperature of a defect-free crystal (even though in cyclic chains crystals some defects will always be present, as the chain can only adopt an extended chain conformation with one fold at each end, as it has no chain ends).

In order to determine $T_{m}^{0}$, the samples were submitted to a specially designed isothermal crystallization procedure from the melt. The samples were first isothermally crystallized (after cooling from the melt at $60^{\circ} \mathrm{C} / \mathrm{min}$ ) for 24 hours at five different crystallization temperatures $\left(T_{c}\right) 90,100,110,120$ and $130{ }^{\circ} \mathrm{C}$. After this time had 
elapsed, the samples were quenched in iced water to prevent any further crystallization during cooling from the melt. To these samples, SAXS measurements were performed at room temperature, as shown in Figure 12a and Figure $12 \mathrm{~b}$ to obtain long periods and from these values lamellar thickness were calculated (see the Supporting Information Figure S4 for the results of PDLA). Additionally, DSC heating scans were performed to the same samples in order to obtain experimental melting points and melting enthalpies.
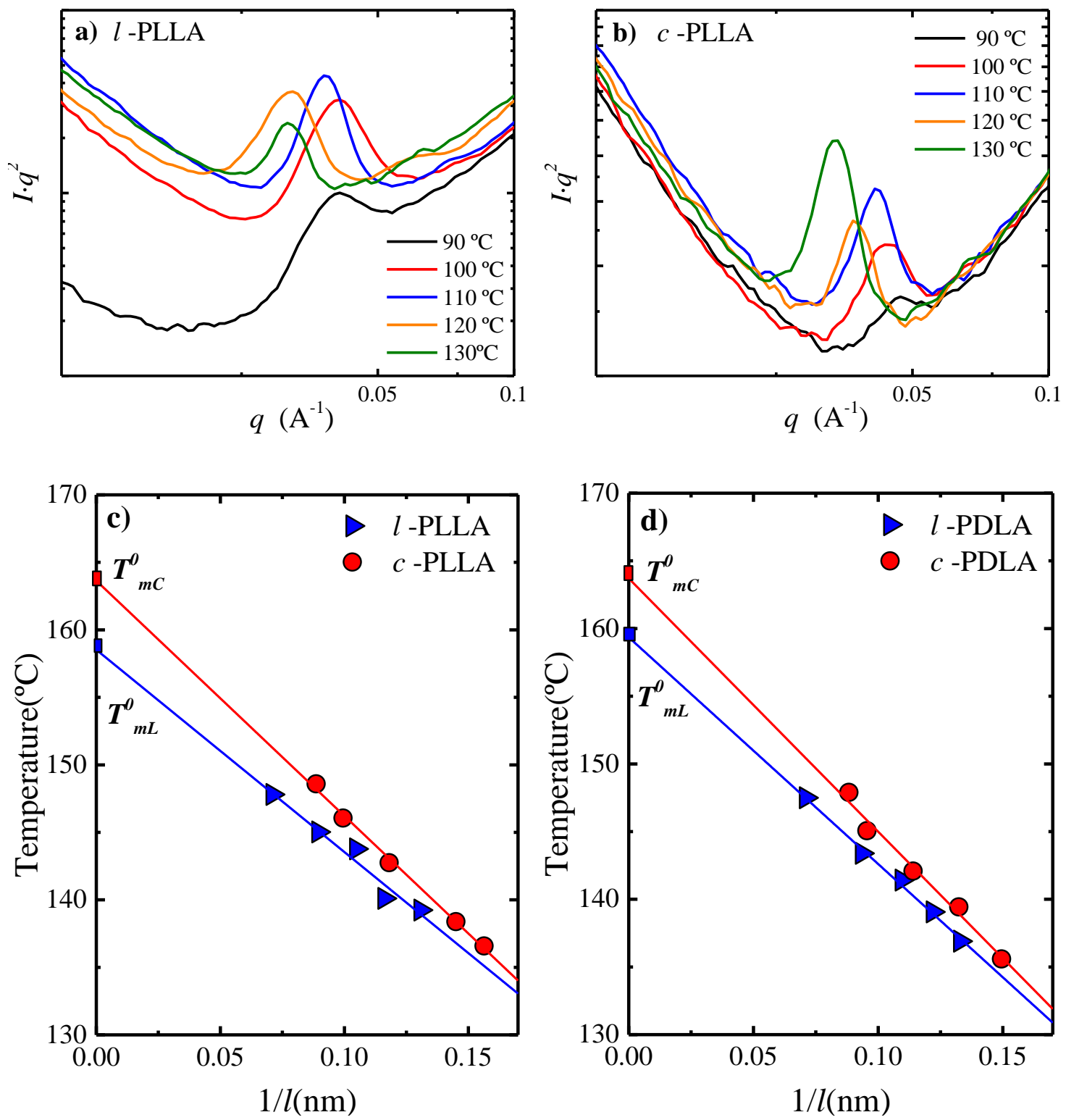

Figure 12. $a$ and $b$ ) Lorentz-corrected SAXS profiles, with intensity times $q^{2}$ as a function of scattering vector. $c$ and d) Representation of the Thompson - Gibbs plots to obtain $T_{m}^{0}$ for linear and cyclic PLA.

From the data presented in Figure $12 \mathrm{a}$ and $12 \mathrm{~b}$, values of $q_{\max }$ were obtained from the peak that is observed in all SAXS profiles. This diffraction signal is due to the 
long period of the lamellar structure in the material and transforming the scattering vector to distance, the long period can be found $(L)$. In order to calculate the value of the lamellar thickness $(l)$, we have employed the approximation $l=X_{v} L$, where $X_{v}$ is the crystal volume fraction (see the Supporting Information for the procedure employed to calculate the crystal volume fractions). ${ }^{62}$ Using the lamellar thickness values and the experimental melting points of the isothermally crystallized samples, the ThompsonGibbs equation can be applied. ${ }^{59}$

Figure 12c and 12d displays the Thompson-Gibbs plots of both cyclic and linear PLA. The equilibrium melting temperatures $\left(T_{m}^{0}\right)$ determined from the intercept are 159 $\pm 1.8{ }^{\circ} \mathrm{C}$ and $159 \pm 0.7^{\circ} \mathrm{C}$ for $l$-PLLA and $l$-PDLA respectively. For $c$-PLLA and $c$ PDLA the $T_{m}^{0}$ values determined are $164 \pm 0.7{ }^{\circ} \mathrm{C}$ and $164 \pm 1.6{ }^{\circ} \mathrm{C}$ respectively. Therefore, $T_{m}^{0}$ is higher in cyclic PLAs than in their linear analogues and D or L stereoregularity has no influence on this thermal property. The determining factor is the topology of the chain. Similar results, where cyclic chains have been found to have higher equilibrium melting points than their linear analogues, have also been reported for $\mathrm{PCL},{ }^{27} \mathrm{PTHF}^{20,32}$ and $\mathrm{PE}^{24}$

The values of the equilibrium melting temperature determined in this work are lower than others reported in the literature. ${ }^{63,64}$ However, it should be noted that $T_{m}^{0}$ values depend on the molecular weight in the low molecular weight range (before it saturates above a critical molecular weight range), a fact confirmed by Pan et al. ${ }^{55}$ specifically for PLLAs. It must be remembered that the samples employed in this work have number average molecular weight values of just $10 \mathrm{~kg} / \mathrm{mol}$, therefore lower apparent melting points and equilibrium melting points are expected in comparison to those exhibited by higher molecular weight samples.

The fold surface free energy $\left(\sigma_{e}\right)$ and cyclization entropy $\left(\Delta S_{c y c}\right)$ can also be obtained from the Thomson-Gibbs analysis based on equations (2) and (3). ${ }^{27}$ The value of $\Delta S_{c y c}$ is $-2 \mathrm{~J} / \mathrm{kgK}$.

The values of $\sigma_{e}$ of linear PLA determined from the slope of the plots are 13 and $15 \mathrm{~mJ} / \mathrm{m}^{2}$ for $l$-PLLA and $l$-PDLA, respectively. Moreover, for cyclic samples 12 and $13 \mathrm{~mJ} / \mathrm{m}^{2}$ values were determined for $c$-PLLA and $c$-PDLA. The differences in the values are within the experimental error of the measurements. The fact that no differences are found may be due to the presence of linear and/or larger cyclic chains impurities in the cyclic chains. Slightly larger $\sigma_{e}$ could be expected for cyclic PLAs as 
larger loops could formed at the crystal surface by the cyclic chains as compared to linear ones, as well as to the presence of the triazole groups that close the cycles and are rejected to the amorphous intervening layers.

It is noted that, for both linear and cyclic PLAs, the values of $\sigma_{e}$ determined from the Thompson-Gibbs analysis in Figure 12c and 12d are smaller than those obtained by us from the Lauritzen-Hoffman analysis (see the Supporting Information). It has been suggested that $\sigma_{e}$ determined by growth rate analysis is a "kinetic" surface free energy, whereas that obtained by the Thompson-Gibbs plot is a "thermodynamic" one. The experimental melting points used in this work for the Thompson-Gibbs plot were determined after the samples have undergone isothermal crystallization for $24 \mathrm{~h}$ and the crystals would have reached their maximum crystal size for the applied supercooling. During the long isothermal crystallization time, the crystal surface may reorganize to reduce the fold surface free energy. On the other hand, the $\sigma_{e}$ obtained from the growth rate is the surface free energy of the crystals just formed during the crystallization process and the great amount of defects on the surface leads to a higher surface free energy. A similar discrepancy has also been found for PCL. ${ }^{27}$

\section{Self-nucleation}

The self-nucleation technique, described in detail in the experimental part, was applied to the four samples employed here, namely, linear and cyclic PLLA and PDLA. Figure 13 shows as examples, the self-nucleation experiments on linear and cyclic PLLAs, where cooling and subsequent heating runs from the applied $T_{s}$ temperatures are shown. 

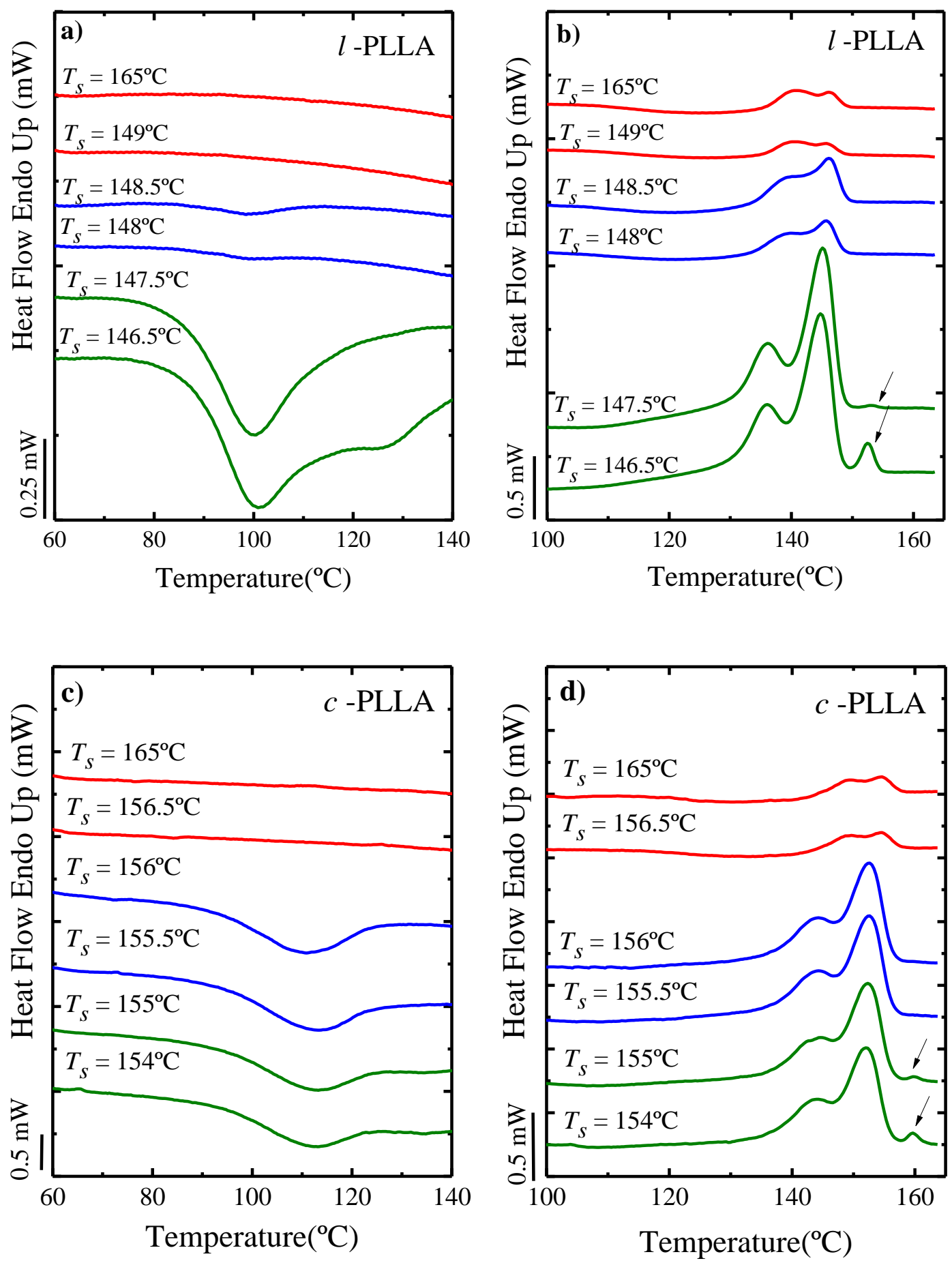

Figure 13. Self-nucleation of l-PLLA and c-PLLA, a) and c) DSC cooling scans from $T_{s}$ and $b$ ) and $\left.d\right)$ the subsequent heating scans.

The self-nucleation process classifies the physical state of the material with respect to its nucleation, in three domains. PLAs cannot crystallize during cooling from the melt at $20^{\circ} \mathrm{C} / \mathrm{min}$. In Figure 13 it can be observed that if $T_{s}$ is greater or equal to 149 ${ }^{\circ} \mathrm{C}$ in $l$-PLLA and $156.5{ }^{\circ} \mathrm{C}$ in $c$-PLLA, no crystallization of the material occurs, 
therefore it is considered that the material is in Domain I or complete melting domain, where the crystalline memory of the material is completely erased and the polymer presents a thermal behaviour identical to that in standards scans.

Figure $13 \mathrm{a}$ and $13 \mathrm{c}$ show that if $T_{s}$ is between $148.5-148{ }^{\circ} \mathrm{C}$ in $l$-PLLA and 156$155.5^{\circ} \mathrm{C}$ in $c$-PLLA an exothermic crystallization peak is observed during cooling. The polymers are now able to crystallize during cooling from the melt thanks to the selfnuclei produced by the thermal protocol applied. This behaviour corresponds to Domain II or self-nucleation domain, since no trace of annealing can be seen in the subsequent melting scans.

Finally, if the selected $T_{s}$ temperature is equal or lower than $147.5^{\circ} \mathrm{C}$ in $l$-PLLA and $155^{\circ} \mathrm{C}$ in $c$-PLLA, partial melting occurs and the unmolten crystals can anneal during the $5 \mathrm{~min}$ at $T_{s}$. In the subsequent heating scans, annealed crystals melt at higher temperatures inducing an extra peak, as observed in Figure 13b and 13d (signalled with arrows). In this $T_{s}$ range, the polymer falls into Domain III. ${ }^{65,66}$ The self-nucleation technique was also applied to PDLA samples and the results are presented in the Supporting Information Figure S5-1.

Table 2. Self-nucleation Domains (DI, DII and DIII) transition temperatures for the indicated samples.

\begin{tabular}{lcc}
\hline & DI to DII $\left({ }^{\circ} \mathbf{C}\right)$ & DII to DIII $\left({ }^{\circ} \mathbf{C}\right)$ \\
\hline$l$-PLLA & 149 & 148 \\
$l$-PDLA & 149 & 148 \\
$c$-PLLA & 156.5 & 155.5 \\
$c$-PDLA & 152.5 & 151.5 \\
\hline
\end{tabular}

After performing the self-nucleation procedure to the samples, the temperatures at which the domain transitions were found are reported in Table 2. The temperatures at which the transition between the different domains occurs are shown on top of the standard DSC heating scans in Figure S5-2 (see Supporting Information).

The temperature range of Domain II in all samples are similar for both linear and cyclic PLAs. In addition, the onset temperature of Domain II occurs in $l$-PLAs at values that are higher than the final melting temperature, as compared to the cyclic ones. The ability to induce self-nucleation when the sample is molten (case of linear samples) is directly related to the crystalline memory of the material. ${ }^{48}$ The crystalline memory effect has been explained by considering that residual chain orientation or 
intermolecular interactions between chains remains (under the applied temperature and time) and prevent the material from achieving an isotropic melt (with random coil conformations). ${ }^{2,28,67}$

Figure S5-2 shows that the crystalline memory of $c$-PLAs are smaller than that exhibited by their linear analogues. This represents another interesting result produced by differences in molecular topology. It is clear that the entanglement density in $l$-PLAs is higher than $c$-PLAs. Therefore, partially disentangled PLA cyclic chains can reach a pseudo-equilibrium random coil conformation much easier than more entangled linear chains. Hence, the crystalline memory of $c$-PLAs can be erased at lower temperatures than in $l$-PLAs (see the DI to DII transition temperatures in Figure S5-2). A similar results has been reported by Perez et al. ${ }^{28}$ for cyclic PCLs.

\section{Successive Self-Nucleation and Annealing (SSA)}

The SSA (Successive Self-nucleation and Annealing technique) was developed by Müller et al. ${ }^{47,49,50}$ Thermal fractionation was applied to the same samples whose self-nucleation domains were determined in the previous section. In order to be able to compare the results, the first self-nucleation temperature for all materials was kept constant at $155.5{ }^{\circ} \mathrm{C}$. This temperature corresponds to the ideal self-nucleation temperature $\left(T_{s(\text { ideal })}\right)$ for the sample with the highest melting point (i.e., $c$-PLLA). A total of 10 cycles were applied, as was explained in the experimental part.

Figure 14a shows the final heating scan after the SSA was performed. Vertical lines indicate the $T_{s}$ values used for the fractionation. All samples show a certain degree of thermal fractionation and therefore a distribution of melting peaks corresponding to various lamellar sizes that melt at different temperatures produced by the SSA procedure. The SSA technique is particularly sensitive to defects that may disrupt the crystallizable sequences. Linear PLAs do not have branches or other defects along the chain, they are expected to be fractionated only by their molecular weight distribution. In the case of cyclic PLAs, the group that binds the chains when the click reaction is made is a defect that can only be located in the amorphous areas of the sample, but it is a single group per molecule, so its impact on molecular fractionation will be reduced. All samples presented in Figure 14a were successfully fractionated.

The melting peaks have been labelled according to their origin. The thermal fraction 1 corresponding to the melting peak 1 and refers to an annealed population produced mainly during the five min holding time at $T_{s}(1)$, although successive steps 
might have also some limited influence in the size of the fraction. Melting peak 2 is produced by $T_{s}(2)$ and so on.
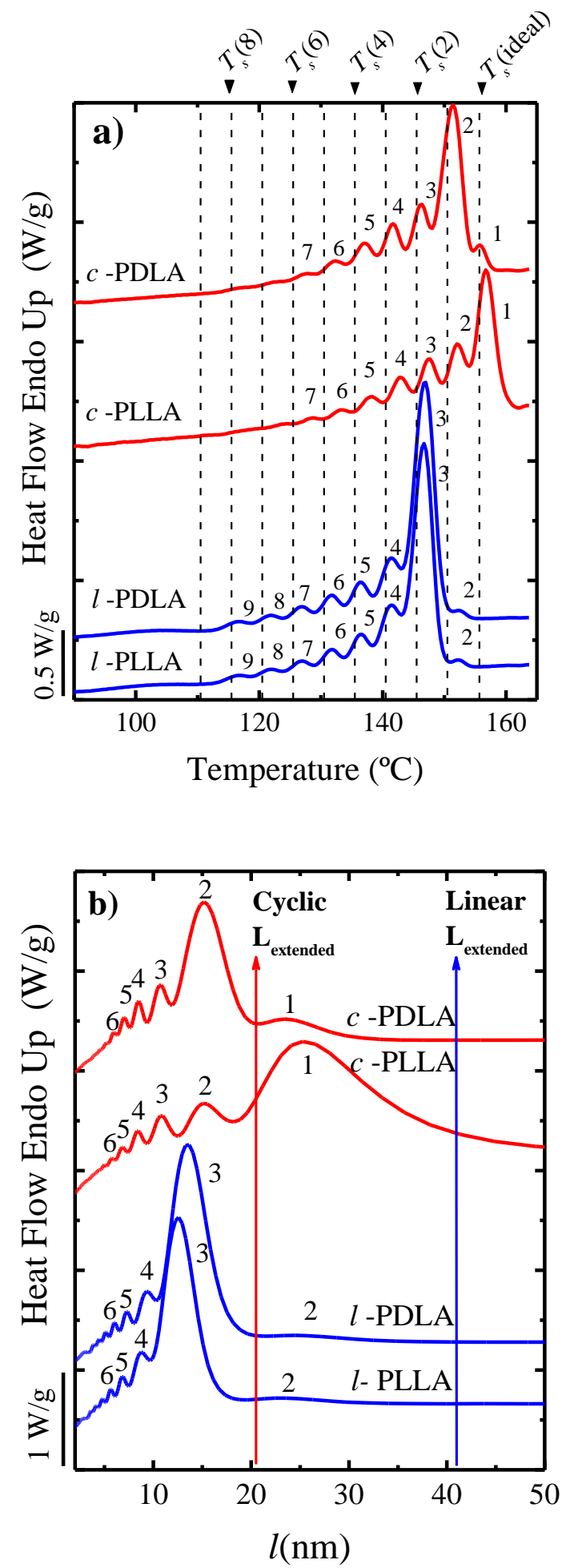

Figure 14. Final heating run after SSA thermal fractionation. a) DSC heating scan after SSA plotted as normalized Heat Flow $(\mathrm{mW} / \mathrm{g}$ ) versus temperature b) Heat Flow $(\mathrm{mW} / \mathrm{g})$ versus lamellar thickness $(\mathrm{l})$ for the indicated samples, $l$ values are calculated from the modified Gibbs-Thomson equation. The vertical lines indicate approximate values of the average extended chain lengths calculated by equation 4. 
It is interesting to observe in Figure 14a that only cyclic samples have the highest melting fraction, or melting peak 1 . Therefore, the temperature $T_{s}(1)$ was only able to cause annealing in the samples with cyclic topology, as they have higher melting points in comparison to their linear analogues. Fraction 2 or melting peak 2 is present in all samples.

SSA experiments show that cyclic PLAs seem to have a higher annealing capacity as compared to their linear analogues, as they are the only ones exhibiting fraction 1. This is remarkable since linear PLA chains could be in theory extended up to a length that would be twice that of a cyclic chain with identical molecular weight. Hence, linear chains have the potential to produce thicker crystals near equilibrium conditions, but under the SSA parameters used (which are dominated by kinetic factors), cyclic PLA crystals apparently anneal more and produce crystals that melt at higher temperatures (compare the highest melting fractions in cyclic and linear materials, i.e., fractions labeled 1 versus fraction labeled 2 respectively). Once again, the lower entanglement density and cyclic chain topology are probably playing an important role to facilitate annealing. Perez et al. ${ }^{28}$ reported similar results for cyclic and linear PCLs.

The extended chain length ( $L_{\text {extended }}$ ) of a linear PLLA chain has been calculated employing eq. (4), derived from crystallographic data for $\alpha$ type crystals:

$$
L_{\text {extended }}=N \times c / 10
$$

where $\mathrm{N}$ is the degree of polymerization, given by $N=M / M_{U R}, M$ is the molecular weight of the sample and $M_{U R}$ is the molecular weight of the repeating unit, 72.06 $\mathrm{g} / \mathrm{mol}$. The parameter $c$ corresponds to the dimension of the crystalline unit cell in the direction of the chain and 10 is the number of repetitive units along the chain axis. From this equation, $L_{\text {extended }}$ can be calculated to be: $41 \mathrm{~nm}$.

On the other hand, in order to estimate the lamellar thickness of the samples after the SSA treatment, their peak melting point values were employed $\left(T_{m}\right)$. By the modified Thomson-Gibbs equation fits obtained in Figure 12, it has been possible to relate these melting temperature values to their respective lamellar thickness values, which are plotted in Figure 14b.

Figure $14 \mathrm{~b}$ shows the distribution of lamellar thickness for both linear and cyclic chains. Cyclic PLA crystals melt at higher temperatures (Figure 14a), but their lamellar 
thicknesses are, according to Figure 14b, in the same range of values (for instance, fractions 1 for cyclics and fraction 2 for linear PLAs are in the same range, between 20 and $30 \mathrm{~nm}$ ). This is due to the different supercooling of the samples, as the ThomsonGibbs equation takes into account the influence of the value of $T_{m}^{0}$, which is different for cyclic and linear PLAs.

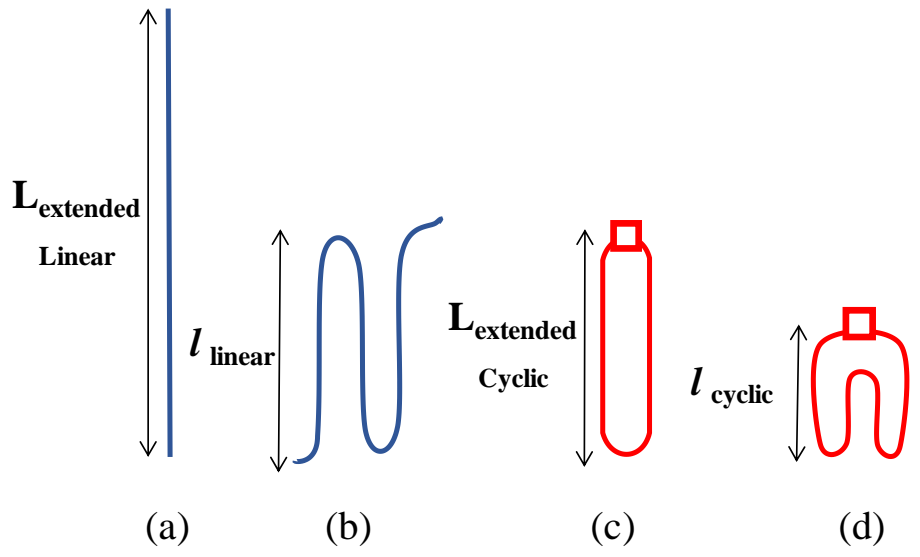

Figure 15. Schematic cartoon illustrating extended and once folded linear and cyclic PLA chains: a) representation of a linear extended chain $\left.\left(L_{\text {extended }}\right) ; b\right)$ once folded linear chain, approximately corresponding to SSA fraction number 2 for l-PLLA; $c$ ) cyclic extended chain $\left(L_{\text {extended }}\right)$ (approximately corresponding to SSA fraction number 1 in c-PLLA) and d) once folded cyclic chain, which approximately corresponds to SSA fraction number 3 for c-PLLA)

The value of the extended chain length $\left(L_{\text {extended }}\right)$ for linear PLLA is indicated with a vertical line in Figure 14b. The equivalent value for the cyclic PLLA chain is also indicated in the Figure 14b, assuming that it will be one half of the calculated value for the linear chain. Therefore, after the SSA treatment, some cyclic chains (those within fraction 1) are most probably extended as a collapsed circle inside the crystal with the folds closely tight in the surface of the crystal, as indicated in the cartoon of Figure 15 .

As indicated in Figure 14b, the length of the extended cyclic chain should be around $20.5 \mathrm{~nm}$ and according to the Thompson-Gibbs equation (which can only give a rough approximation to lamellar thickness values), after SSA, cyclic chains have fraction number 1 with lamellar thickness in the range between $20-30 \mathrm{~nm}$. So within the errors involved (approximations of the Thompson-Gibbs equation and mean values of molecular weight employed to calculate the extended chain values), it seems that a certain fraction of cyclic chains may crystallize with extended chains (like in Figure $15 c)$. 
On the other hand, according to the obtained value for the extended linear chains, they should crystallize in fraction 2 (the highest melting fraction) with at least one fold, not being able to extend fully (see Figure 15b). The lower density of entanglements of cyclic chains probably plays an important role in facilitating the full extension of a certain fraction of the chains. ${ }^{15}$

\section{Conclusions}

In the present paper, we studied for the first time the influence of chain topology and stereochemistry on the nucleation and crystallization kinetics of PLAs. WAXS results demonstrated that chain topology has no effect on the crystal structure of both PDLA and PLLA. On the other hand, important differences in nucleation and crystallization kinetics were found, as cyclic chains nucleate much faster than linear ones. The effect of nucleation dominated the overall crystallization, as the growth rate was influenced by the presence of high molecular weight "impurities" present in the cyclic samples.

The differences found between cyclic and linear PLA chains in terms of thermal, nucleation and overall crystallization kinetics are due to both kinetic and thermodynamic effects. The kinetic effects are related to the faster diffusion of cyclic chains which stem from their lack of chain ends (that reduce entanglement density) and more collapsed compact coil conformations. The thermodynamic effects are due to the enhanced supercooling of the cyclic chains in comparison to linear ones, as the equilibrium melting point of cyclic chains was found to be higher than that of linear chains as a consequence of the lower entropy of cyclic chains in the melt.

The reduced density of entanglements of cyclic PLA chains induces a shorter crystalline memory in comparison with $l$-PLAs. SSA results show that $c$-PLAs have a higher annealing capacity than linear chains and form fractions that can melt at higher temperatures in comparison to $l$-PLAs. In fact, the highest melting point fractions in $c$ PLAs formed extended chain cyclic molecules, while in $l$-PLAs, the highest melting point fractions are formed by folded chains.

The effect of chain topology was found to be dominant and more important than that of stereochemistry, as the results for PLLA and PDLA were in several cases very similar. 


\section{Acknowledgments}

The UPV/EHU team would like to acknowledge funding from the following projects: "UPV/EHU Infrastructure: INF 14/38"; "Mineco/FEDER: SINF 130I001726XV1/Ref: UNPV13-4E-1726" and "Mineco MAT2014-53437-C2-P", 'Ministerio de Economia y Competitividad (MINECO), code: MAT2015-63704-P (MINECO/FEDER, UE) and by the Eusko Jaurlaritza (Basque Government), code: IT654-13. O.C acknowledges financial support from the European Commission and Région Wallonne FEDER program (Materia Nova) and OPTI²MAT program of excellence, by the Interuniversity Attraction Pole Program (P7/05) initiated by the Belgian Science Policy office and by the FNRS-FRFC. OC is Research Associate of the F.R.S.-FNRS. Organic Synthesis and Mass Spectrometry Laboratory thanks F.R.S.FNRS for the financial support for the acquisition of the Waters QToF Premier and Synapt-G2Si mass spectrometers and for continuing support. Finally, all authors would like to acknowledge Research and Innovation Staff Exchange (RISE) H2020-MSCARISE-2017-778092, project BIODEST for promoting cooperation between the Mons team and the UPV/EHU team.

\section{Supporting Information}

S1. Complementary data on nucleation measurements performed by Polarized Light Optical Microscopy. S2. Lauritzen and Hoffman crystallization theory. S3. Fitting of DSC isothermal data to the Avrami model. S4. Complementary SAXS data and Crystal volume fractions and S5. Complementary data on self-nucleation. 


\section{References}

(1) Beckham, H. W., Ring Polymers: Effective Isolation and Unique Properties. In Complex Macromolecular Architectures: Synthesis, Characterization, and SelfAssembly, Hadjichristidis, N., Hirao, A., Tezuka, Y., Du Prez, F., 2011; p 791-821.

(2) Perez-Camargo R.A; Mugica A; Zubitur M; Müller A.J. Crystallization of Cyclic Polymers. Adv. Polym. Sci. 2017, 276, 93-132.

(3) Schappacher, M.; Deffieux, A. Controlled synthesis of bicyclic 'eight-shaped' poly(chloroethyl vinyl ether)s. Macromolecules 1995, 28 (8), 2629-2636.

(4) Kubo, M.; Hayashi, T.; Kobayashi, H.; Itoh, T. Syntheses of tadpole- and eightshaped polystyrenes using cyclic polystyrene as a building block. Macromolecules 1998, 31 (4), 1053-1057.

(5) Oike, H.; Washizuka, M.; Tezuka, Y. Designing an "A-ring-with-branches" polymer topology by electrostatic self-assembly and covalent fixation with interiorly functionalized telechelics having cyclic ammonium groups. Macromol. Rapid Commun. 2001, 22 (14), 1128-1134.

(6) Tezuka, Y.; Komiya, R.; Washizuka, M. Designing 8-shaped polymer topology by metathesis condensation with cyclic poly(THF) precursors having allyl groups. Macromolecules 2003, 36 (1), 12-17.

(7) Jia, Z.; Fu, Q.; Huang, J. Synthesis of amphiphilic macrocyclic graft copolymer consisting of a poly(ethylene oxide) ring and multi-polystyrene lateral chains. Macromolecules 2006, 39 (16), 5190-5193.

(8) Fan, X.; Huang, B.; Wang, G.; Huang, J. Synthesis of amphiphilic heteroeightshaped polymer cyclic -[poly(ethylene oxide)- b -polystyrene $]_{2}$ via "click" chemistry. Macromolecules 2012, 45 (9), 3779-3786.

(9) Wang, G.; Fan, X.; Hu, B.; Zhang, Y.; Huang, J. Synthesis of eight-shaped poly(ethylene oxide) by the combination of glaser coupling with ring-opening polymerization. Macromol. Rapid Commun. 2011, 32 (20), 1658-1663.

(10) Laurent, B. A.; Grayson, S. M. Synthesis of cyclic dendronized polymers via divergent "graft- from" and convergent click "graft-to" routes: Preparation of modular toroidal macromolecules. J. Am. Chem. Soc. 2011, 133 (34), 13421-13429.

(11) Shi, G. Y.; Pan, C. Y. Synthesis of well-defined figure-of-eight-shaped polymers by a combination of ATRP and click chemistry. Macromol. Rapid Commun. 2008, 29 (20), 1672-1678.

(12) Semlyen, J. A. Cyclic polymers. Pure Appl. Chem. 1981, 53 (9), 1797-1804.

(13) Kricheldorf, H. R. Cyclic Polymers: Synthetic strategies and physical properties. $J$. Polym. Sci. Part A: Polym. Chem. 2010, 48 (2), 251-284.

(14) Nam, S.; Leisen, J.; Breedveld, V.; Beckham, H. W. Dynamics of unentangled cyclic and linear poly(oxyethylene) melts. Polymer 2008, 49 (25), 5467-5473.

(15) Xiao, H.; Luo, C.; Yan, D.; Sommer, J.-U. Molecular Dynamics Simulation of Crystallization Cyclic Polymer Melts As Compared to Their Linear Counterparts. Macromolecules 2017, 50 (24), 9796-9806.

(16) Lee, K. S.; Wegner, G.; Hsu, S. L. Vibrational spectroscopic studies of linear and cyclic alkanes $\mathrm{C}_{\mathrm{n}} \mathrm{H}_{2 \mathrm{n}+2}, \mathrm{C}_{\mathrm{n}} \mathrm{H}_{2 \mathrm{n}}$ with $24 \leq \mathrm{n} \leq 288$ : Chain folding, chain packing and conformations. Polymer 1987, 28 (6), 889-896.

(17) Yang, Z.; Yu, G. E.; Cooke, J.; Ali-Adib, Z.; Viras, K.; Matsuura, H.; Ryan, A. J.; Booth, C. Preparation and crystallinity of a large unsubstituted crown ether, cyclic heptacosa(oxyethylene) (cyclo-E27, 81-crown-27), studied by Raman spectroscopy, Xray scattering and differential scanning calorimetry. J. Chem. Soc., Faraday Trans. 1996, 92 (17), 3173-3182. 
(18) Cooke, J.; Viras, K.; Yu, G. E.; Sun, T.; Yonemitsu, T.; Ryan, A. J.; Price, C.; Booth, C. Large cyclic poly(oxyethylene)s: Chain folding in the crystalline state studied by Raman spectroscopy, $\mathrm{x}$-ray scattering, and differential scanning calorimetry. Macromolecules 1998, 31 (9), 3030-3039.

(19) Yu, G. E.; Sun, T.; Yan, Z. G.; Price, C.; Booth, C.; Cook, J.; Ryan, A. J.; Viras, K. Low-molar-mass cyclic poly(oxyethylene)s studied by Raman spectroscopy, X-ray scattering and differential scanning calorimetry. Polymer 1997, 38 (1), 35-42.

(20) Tezuka, Y.; Ohtsuka, T.; Adachi, K.; Komiya, R.; Ohno, N.; Okui, N. A defect-free ring polymer: Size-controlled cyclic poly(tetrahydrofuran) consisting exclusively of the monomer unit. Macromol. Rapid Commun. 2008, 29 (14), 1237-1241.

(21) Córdova, M. E.; Lorenzo, A. T.; Müller, A. J.; Hoskins, J. N.; Grayson, S. M. A comparative study on the crystallization behavior of analogous linear and cyclic poly $(\varepsilon-$ caprolactones). Macromolecules 2011, 44 (7), 1742-1746.

(22) Schäler, K.; Ostas, E.; Schröter, K.; Thurn-Albrecht, T.; Binder, W. H.; Saalwächter, K. Influence of chain topology on polymer dynamics and crystallization. investigation of linear and cyclic poly( $\varepsilon$-caprolactone)s by $1 \mathrm{H}$ solid-state NMR methods. Macromolecules 2011, 44 (8), 2743-2754.

(23) Shin, E. J.; Jeong, W.; Brown, H. A.; Koo, B. J.; Hedrick, J. L.; Waymouth, R. M. Crystallization of cyclic polymers: Synthesis and crystallization behavior of high molecular weight cyclic poly(E-caprolactone)s. Macromolecules 2011, 44 (8), 27732779.

(24) Kitahara, T.; Yamazaki, S.; Kimura, K. Effects of topological constraint and knot entanglement on the crystal growth of polymers proved by growth rate of spherulite of cyclic polyethylene. Kobunshi Ronbunshu 2011, 68 (10), 694-701.

(25) Takeshita, H.; Shiomi, T., Crystallization of cyclic and branched polymers. In Topological Polymer Chemistry: Progress of Cyclic Polymers in Syntheses, Properties, and Functions, 2012; p 317-328.

(26) Shin, E. J.; Jones, A. E.; Waymouth, R. M. Stereocomplexation in cyclic and linear polylactide blends. Macromolecules 2012, 45 (1), 595-598.

(27) Su, H. H.; Chen, H. L.; Díaz, A.; Casas, M. T.; Puiggalí, J.; Hoskins, J. N.; Grayson, S. M.; Pérez, R. A.; Müller, A. J. New insights on the crystallization and melting of cyclic PCL chains on the basis of a modified Thomson-Gibbs equation. Polymer 2013, 54 (2), 846-859.

(28) Pérez, R. A.; Córdova, M. E.; López, J. V.; Hoskins, J. N.; Zhang, B.; Grayson, S. M.; Müller, A. J. Nucleation, crystallization, self-nucleation and thermal fractionation of cyclic and linear poly(E-caprolactone)s. React. Funct. Polym. 2014, 80 (1), 71-82.

(29) Pérez, R. A.; López, J. V.; Hoskins, J. N.; Zhang, B.; Grayson, S. M.; Casas, M. T.; Puiggalí, J.; Müller, A. J. Nucleation and antinucleation effects of functionalized carbon nanotubes on cyclic and linear Poly(E-caprolactones). Macromolecules 2014, 47 (11), 3553-3566.

(30) Wang, J.; Li, Z.; Pérez, R. A.; Müller, A. J.; Zhang, B.; Grayson, S. M.; Hu, W. Comparing crystallization rates between linear and cyclic poly(epsilon-caprolactones) via fast-scan chip-calorimeter measurements. Polymer 2015, 63, 34-40.

(31) Sugai, N.; Asai, S.; Tezuka, Y.; Yamamoto, T. Photoinduced topological transformation of cyclized polylactides for switching the properties of homocrystals and stereocomplexes. Polym. Chem. 2015, 6 (19), 3591-3600.

(32) Takeshita, H.; Poovarodom, M.; Kiya, T.; Arai, F.; Takenaka, K.; Miya, M.; Shiomi, T. Crystallization behavior and chain folding manner of cyclic, star and linear poly(tetrahydrofuran)s. Polymer 2012, 53 (23), 5375-5384. 
(33) Li, H.; Jéröme, R.; Lecomte, P. Synthesis of tadpole-shaped copolyesters based on living macrocyclic poly( $\varepsilon$-caprolactone). Polymer 2006, 47 (26), 8406-8413.

(34) Lee, K. S.; Wegner, G. Linear and cyclic alkanes $\left(\mathrm{C}_{\mathrm{n}} \mathrm{H}_{2 n+2}, \mathrm{CNH}_{2 n}\right)$ with $\mathrm{n}>100$. Synthesis and evidence for chain-folding. Macromol. Rapid Commun. 1985, 6 (3), 203208.

(35) Bielawski, C. W.; Benitez, D.; Grubbs, R. H. An "endless" route to cyclic polymers. Science 2002, 297 (5589), 2041-2044.

(36) Lopez, J. V.; Perez-Camargo, R. A.; Zhang, B.; Grayson, S. M.; Muller, A. J. The influence of small amounts of linear polycaprolactone chains on the crystallization of cyclic analogue molecules. RSC Adv. 2016, 6 (53), 48049-48063.

(37) Sugai, N.; Yamamoto, T.; Tezuka, Y. Synthesis of orientationally isomeric cyclic stereoblock polylactides with head-to-head and head-to-tail linkages of the enantiomeric segments. ACS Macro Lett. 2012, 1 (7), 902-906.

(38) Yang, J.; Wang, Y.; Rassat, A.; Zhang, Y.; Sinaÿ, P. Synthesis of novel highly water-soluble 2:1 cyclodextrin/fullerene conjugates involving the secondary rim of $\beta$ cyclodextrin. Tetrahedron 2004, 60 (52), 12163-12168.

(39) Malkoch, M.; Schleicher, K.; Drockenmuller, E.; Hawker, C. J.; Russell, T. P.; Wu, P.; Fokin, V. V. Structurally Diverse Dendritic Libraries: A Highly Efficient Functionalization Approach Using Click Chemistry. Macromolecules 2005, 38 (9), 3663-3678.

(40) Josse, T.; De Winter, J.; Dubois, P.; Coulembier, O.; Gerbaux, P.; Memboeuf, A. A tandem mass spectrometry-based method to assess the architectural purity of synthetic polymers: a case of a cyclic polylactide obtained by click chemistry. Polym. Chem. 2015, 6 (1), 64-69.

(41) Liénard, R.; Josse, T.; De Winter, J.; Dubois, F.; Gerbaux, P.; Coulembier, O. Preparation of highly pure cyclo-polylactides by optimization of the copper-catalyzed azide-alkyne cycloaddition reaction. Polimery 2017, 62, (4), 283-290.

(42) Hoskins, J. N.; Grayson, S. M. Synthesis and Degradation Behavior of Cyclic Poly(epsilon-caprolactone). Macromolecules 2009, 42 (17), 6406-6413.

(43) Lorenzo, A. T.; Arnal, M. L.; Albuerne, J.; Müller, A. J. DSC isothermal polymer crystallization kinetics measurements and the use of the Avrami equation to fit the data: Guidelines to avoid common problems. Polym. Test. 2007, 26 (2), 222-231.

(44) Quero, E.; Müller, A. J.; Signori, F.; Coltelli, M. B.; Bronco, S. Isothermal coldcrystallization of PLA/PBAT blends with and without the addition of acetyl tributyl citrate. Macromol. Chem. Phys. 2012, 213 (1), 36-48.

(45) Liao, R.; Yang, B.; Yu, W.; Zhou, C. Isothermal cold crystallization kinetics of polylactide/nucleating agents. J J. Appl. Polym. Sci. 2007, 104 (1), 310-317.

(46) Fillon, B.; Wittmann, J. C.; Lotz, B.; Thierry, A. Self-nucleation and recrystallization of isotactic polypropylene ( $\alpha$ phase) investigated by differential scanning calorimetry. J. Polym. Sci., Part B: Polym. Phys. 1993, 31 (10), 1383-1393.

(47) Müller, A. J.; Arnal, M. L. Thermal fractionation of polymers. Prog. Polym. Sci. 2005, 30 (5), 559-603.

(48) Lorenzo, A. T.; Arnal, M. L.; Sánchez, J. J.; Müller, A. J. Effect of annealing time on the self-nucleation behavior of semicrystalline polymers. J J. Polym. Sci., Part B: Polym. Phys. 2006, 44 (12), 1738-1750.

(49) Müller, A. J.; Hernández, Z. H.; Arnal, M. L.; Sánchez, J. J. Successive selfnucleation/annealing (SSA): A novel technique to study molecular segregation during crystallization. Polym. Bull. 1997, 39 (4), 465-472. 
(50) Müller, A. J.; Michell, R. M.; Pérez, R. A.; Lorenzo, A. T. Successive Selfnucleation and Annealing (SSA): Correct design of thermal protocol and applications. Eur. Polym. J. 2015, 65 (Supplement C), 132-154.

(51) Li, Y.; Hoskins, J. N.; Sreerama, S. G.; Grayson, S. M. MALDI-TOF Mass Spectral Characterization of Polymers Containing an Azide Group: Evidence of Metastable Ions. Macromolecules 2010, 43 (14), 6225-6228.

(52) Sarasua, J.-R.; Prud'homme, R. E.; Wisniewski, M.; Le Borgne, A.; Spassky, N. Crystallization and Melting Behavior of Polylactides. Macromolecules 1998, 31 (12), 3895-3905.

(53) Wang, Y.; Mano, J. F. Influence of melting conditions on the thermal behaviour of poly(l-lactic acid). Eur. Polym. J. 2005, 41 (10), 2335-2342.

(54) Kobayashi, J.; Asahi, T.; Ichiki, M.; Oikawa, A.; Suzuki, H.; Watanabe, T.; Fukada, E.; Shikinami, Y. Structural and optical properties of poly lactic acids. J. Appl. Phys. 1995, 77 (7), 2957-2973.

(55) Pan, P.; Kai, W.; Zhu, B.; Dong, T.; Inoue, Y. Polymorphous Crystallization and Multiple Melting Behavior of Poly(l-lactide): Molecular Weight Dependence. Macromolecules 2007, 40 (19), 6898-6905.

(56) Hur, K.; Winkler, R. G.; Yoon, D. Y. Comparison of ring and linear polyethylene from molecular dynamics simulations. Macromolecules 2006, 39 (12), 3975-3977.

(57) Suzuki, J.; Takano, A.; Matsushita, Y. Topological effect in ring polymers investigated with Monte Carlo simulation. J. Chem. Phys. 2008, 129 (3), 034903.

(58) Schultz, J. M., In Polymer Crystallization: The Development of Crystalline Order in Thermoplastic Polymers., Eds. Society A. C.; 2001.

(59) Gedde, U. W. In Polymer physics., Eds. Springer Science \& Business Media, 1995.

(60) Strobl, G., A Multiphase Model Describing Polymer Crystallization and Melting. In Progress in Understanding of Polymer Crystallization, Reiter, G., Strobl, G. R., Eds. Springer Berlin Heidelberg, 2007; p 481-502.

(61) Avrami, M. Granulation, Phase Change, and Microstructure Kinetics of Phase Change. III. J. Chem. Phys. 1941, 9, 177-184.

(62) Zaldua, N.; Mugica, A.; Zubitur, M.; Iturrospe, A.; Arbe, A.; Re, G. L.; Raquez, J.M.; Dubois, P.; Muller, A. J. The role of PLLA-g-montmorillonite nanohybrids in the acceleration of the crystallization rate of a commercial PLA. CrystEngComm 2016, 18 (48), 9334-9344.

(63) Muller, A. J.; Avila, M.; Saenz, G.; Salazar, J., CHAPTER 3 Crystallization of PLA-based Materials. In Poly(lactic acid) Science and Technology: Processing, Properties, Additives and Applications, Jimenez. A., Peltzer. M., Ruseckaite. R., Eds. The Royal Society of Chemistry: 2015; p 66-98.

(64) Saeidlou, S.; Huneault, M. A.; Li, H.; Park, C. B. Poly(lactic acid) crystallization. Prog. Polym. Sci. 2012, 37 (12), 1657-1677.

(65) Fillon, B.; Lotz, B.; Thierry, A.; Wittmann, J. C. Self-nucleation and enhanced nucleation of polymers. Definition of a convenient calorimetric "efficiency scale" and evaluation of nucleating additives in isotactic polypropylene ( $\alpha$ phase). J. Polym. Sci., Part B: Polym. Phys. 1993, 31 (10), 1395-1405.

(66) Fillon, B.; Thierry, A.; Wittmann, J. C.; Lotz, B. Self-nucleation and recrystallization of polymers. Isotactic polypropylene, $\beta$ phase: $\beta-\alpha$ conversion and $\beta-\alpha$ growth transitions. J. Polym. Sci., Part B: Polym. Phys. 1993, 31 (10), 1407-1424.

(67) Sangroniz, L.; Cavallo, D.; Santamaria, A.; Müller, A. J.; Alamo, R. G. Thermorheologically Complex Self-Seeded Melts of Propylene-Ethylene Copolymers. Macromolecules 2017, 50 (2), 642-651. 


\section{TABLE OF CONTENTS (TOC) GRAPHIC}

\section{Influence of chain topology (cyclic versus linear) on the nucleation and isothermal crystallization of poly(L-lactide) and poly(D-lactide)}

\footnotetext{
${ }^{1}$ Nerea Zaldua, ${ }^{2,3}$ Romain Liénard, ${ }^{2}$ Thomas Josse, ${ }^{4}$ Manuela Zubitur, ${ }^{1}$ Agurtzane Mugica, ${ }^{5}$ Amaia Iturrospe, ${ }^{5}$ Arantxa Arbe, ${ }^{3}$ Julien De Winter, ${ }^{2}$ Olivier Coulembier*, ${ }^{1,6}$ Alejandro J. Müller*
}

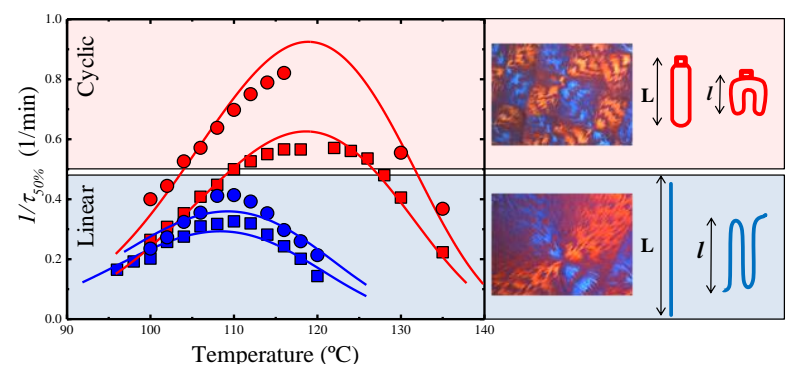

